\title{
DECOUPLED SCHEMES FOR A NON-STATIONARY MIXED STOKES-DARCY MODEL
}

\author{
MO MU AND XIAOHONG ZHU
}

\begin{abstract}
We study numerical methods for solving a non-stationary mixed Stokes-Darcy problem that models coupled fluid flow and porous media flow. A decoupling approach based on interface approximation via temporal extrapolation is proposed for devising decoupled marching algorithms for the mixed model. Error estimates are derived and numerical experiments are conducted to demonstrate the computational effectiveness of the decoupling approach.
\end{abstract}

\section{INTRODUCTION}

A wide variety of real world applications involve different physical processes in different regions of a simulation domain. We are interested in the coupling between the surface and subsurface motion of a fluid, where the behavior of the fluid can be described by different partial differential equations, such as the free surface NavierStokes equations, the Navier-Stokes equations, or the Stokes equations in the surface region, and Darcy's law in the subsurface region [2, 6, 17, 23. These coupled models find important applications in practice, for instance the simulation of flooding in dry areas. When further coupled with transport-diffusion equations, they can also be applied to study the propagation and diffusion of pollutants dispersed in water. In this paper, we focus on the coupled fluid flow and porous media flow modeled by a mixed Stokes-Darcy problem. There is a rich literature on the mathematical analysis, numerical methods and applications for the mixed Stokes-Darcy model; see, e.g., 1, 6, 7, 8, 9, 13, 14, 15, 16, 20, and the references therein.

Specifically, let us consider a fluid flow in $\Omega_{f}$ coupled with a porous media flow in $\Omega_{p}$, see Figure1, where $\Omega_{f}, \Omega_{p} \subset \mathbb{R}^{d}(d=2$ or 3$)$ are bounded domains, $\Omega_{f} \cap \Omega_{p}=\emptyset$, and $\bar{\Omega}_{f} \cap \bar{\Omega}_{p}=\Gamma$. Denote by $\bar{\Omega}=\bar{\Omega}_{f} \cup \bar{\Omega}_{p}, \mathbf{n}_{f}$ and $\mathbf{n}_{p}$ the unit outward normal vectors on $\partial \Omega_{f}$ and $\partial \Omega_{p}$, respectively, and $\boldsymbol{\tau}_{i}, i=1, \ldots, d-1$, the unit tangential vectors on the interface $\Gamma$. Note that $\mathbf{n}_{p}=-\mathbf{n}_{f}$ on $\Gamma$.

Received by the editor October 2, 2008 and, in revised form, May 19, 2009.

2000 Mathematics Subject Classification. Primary 65N15, 65N30, 76D07, 76S05.

Key words and phrases. Mixed fluid flow and porous media flow, Stokes equations, Darcy's law, interface coupling, decoupled numerical methods.

The first author was supported in part by Hong Kong RGC Competitive Earmarked Research Grant HKUST601907.

The second author was supported in part by Hong Kong RGC Competitive Earmarked Research Grant HKUST601907. 


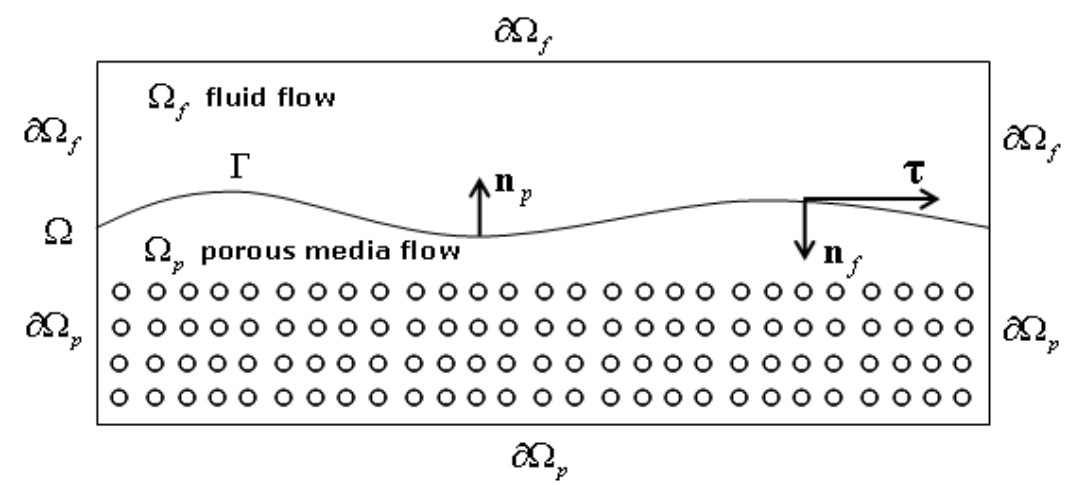

FIgURE 1. The global domain $\Omega$ consisting of the fluid region $\Omega_{f}$ and the porous media region $\Omega_{p}$, separated by the interface $\Gamma$.

Let $T>0$ be a finite time. The fluid flow is governed by the Stokes equations:

$$
\begin{aligned}
\frac{\partial \mathbf{u}_{f}}{\partial t}-\nu \Delta \mathbf{u}_{f}+\nabla p_{f}=\mathbf{g}_{f} & \text { in } \Omega_{f} \times(0, T], \\
\nabla \cdot \mathbf{u}_{f}=0 & \text { in } \Omega_{f} \times(0, T], \\
\mathbf{u}_{f}(\mathbf{x}, 0)=\mathbf{u}_{f}^{0}(\mathbf{x}) & \text { in } \Omega_{f},
\end{aligned}
$$

where $\mathbf{u}_{f}(\mathbf{x}, t)$ represents the velocity of the fluid flow in $\Omega_{f}, p_{f}(\mathbf{x}, t)$ the kinetic pressure, $\mathbf{g}_{f}$ the external force, and $\nu>0$ the kinematic viscosity.

The porous media flow is governed by the following equations [2, 23]:

$$
\begin{aligned}
S_{0} \frac{\partial \varphi}{\partial t}+\nabla \cdot \mathbf{q}=g_{p} & \text { in } \Omega_{p} \times(0, T], \\
\mathbf{q}=-\mathbf{K} \nabla \varphi & \text { in } \Omega_{p} \times(0, T] \text { (Darcy's law), } \\
\mathbf{u}_{p}=\frac{\mathbf{q}}{n} & \text { in } \Omega_{p} \times(0, T], \\
\varphi(\mathbf{x}, 0)=\varphi^{0}(\mathbf{x}) & \text { in } \Omega_{p},
\end{aligned}
$$

where $\varphi(\mathbf{x}, t)$ is the piezometric head, $\mathbf{q}$ is the specific discharge defined as the volume of the fluid flowing per unit time through a unit cross-sectional area normal to the direction of the flow, $\mathbf{u}_{p}$ is the fluid velocity in $\Omega_{p}, S_{0}$ is the specific mass storativity coefficient, $\mathbf{K}$ is the hydraulic conductivity tensor, $n$ is the volumetric porosity, and $g_{p}$ is the source term. Note that $\varphi=z+\frac{p_{p}}{\rho g}$, the sum of elevation head plus pressure head, where $p_{p}$ is the pressure of the fluid in $\Omega_{p}, \rho$ is the density of the fluid, $g$ is the gravitational acceleration, and $z$ is the elevation from a reference level. Without loss of generality, we assume $z=0$. Furthermore, we assume that $\mathbf{K}=\operatorname{diag}(K, \ldots, K)$ with $K \in L^{\infty}\left(\Omega_{p}\right), K>0$, which implies that the porous media is homogeneous. Finally, by using Darcy's law (1.5), the continuity equation (1.4) in $\Omega_{p}$ can be written in the parabolic form

$$
S_{0} \frac{\partial \varphi}{\partial t}-\nabla \cdot(\mathbf{K} \nabla \varphi)=g_{p} \quad \text { in } \Omega_{p} \times(0, T] .
$$

A key part in a mixed model is the interface coupling conditions. For the StokesDarcy model, the following interface conditions have been extensively studied and 
used in the literature:

$$
\begin{array}{rr}
\mathbf{u}_{f} \cdot \mathbf{n}_{f}+\mathbf{u}_{p} \cdot \mathbf{n}_{p}=0 & \text { on } \Gamma \times(0, T], \\
p_{f}-\nu \mathbf{n}_{f} \frac{\partial \mathbf{u}_{f}}{\partial \mathbf{n}_{f}}=\rho g \varphi & \text { on } \Gamma \times(0, T], \\
-\nu \boldsymbol{\tau}_{i} \frac{\partial \mathbf{u}_{f}}{\partial \mathbf{n}_{f}}=\frac{\alpha}{\sqrt{\boldsymbol{\tau}_{i} \cdot \mathbf{K} \boldsymbol{\tau}_{i}}}\left(\mathbf{u}_{f}-\mathbf{u}_{p}\right) \cdot \boldsymbol{\tau}_{i}, & \\
i=1, \ldots, d-1 & \text { on } \Gamma \times(0, T],
\end{array}
$$

where $\alpha$ is a positive parameter depending on the properties of the porous medium and must be experimentally determined. The first interface condition (1.9) ensures the mass conservation across the interface $\Gamma$, and using (1.5) and (1.6), it can be rewritten as

$$
\mathbf{u}_{f} \cdot \mathbf{n}_{f}=\frac{\mathbf{K}}{n} \frac{\partial \varphi}{\partial \mathbf{n}_{p}} \quad \text { on } \Gamma \times(0, T] .
$$

The second condition (1.10) is the balance of the normal forces across the interface.

The third one (1.11) states that the slip velocity along the interface is proportional to the shear stress along the interface. There have been many discussions on this slip condition. It is even unclear if this third condition leads to a well-posed problem. However, it has been observed that in practice the term $\mathbf{u}_{p} \cdot \boldsymbol{\tau}_{i}$ on the right hand side from the porous media flow is much smaller than other terms and is thus negligible. This leads to the most accepted interface condition, known as the Beavers-Joseph-Saffman law [3, 13, 14, 19, 21]:

$$
-\nu \boldsymbol{\tau}_{i} \frac{\partial \mathbf{u}_{f}}{\partial \mathbf{n}_{f}}=\frac{\alpha}{\sqrt{\boldsymbol{\tau}_{i} \cdot \mathbf{K} \boldsymbol{\tau}_{i}}} \mathbf{u}_{f} \cdot \boldsymbol{\tau}_{i}, i=1, \ldots, d-1 \quad \text { on } \Gamma \times(0, T],
$$

which will be used in the rest of the paper.

Several types of boundary conditions for this coupled model are discussed in [6]. For simplicity, in this paper we assume the homogeneous Dirichlet boundary conditions for the coupled model, that is,

$$
\begin{aligned}
\mathbf{u}_{f}=\mathbf{0} & \text { on } \partial \Omega_{f} \backslash \Gamma, \\
\phi=0 & \text { on } \partial \Omega_{p} \backslash \Gamma .
\end{aligned}
$$

Denote $W=H_{f} \times H_{p}$ and $Q=L^{2}\left(\Omega_{f}\right)$, where

$$
H_{f}=\left\{\mathbf{v} \in\left(H^{1}\left(\Omega_{f}\right)\right)^{d}: \mathbf{v}=0 \text { on } \partial \Omega_{f, D}\right\}
$$

and

$$
H_{p}=\left\{\psi \in H^{1}\left(\Omega_{p}\right): \psi=0 \text { on } \partial \Omega_{p, D}\right\} .
$$

The space $L^{2}(D)$, where $D=\Omega_{f}$ or $\Omega_{p}$, is equipped with the usual $L^{2}$-scalar product $(\cdot, \cdot)$ and $L^{2}$-norm $\|\cdot\|_{L^{2}} \triangleq\|\cdot\|_{0}$. The spaces $H_{f}$ and $H_{p}$ are equipped with the following norms:

$$
\begin{aligned}
\|\mathbf{u}\|_{H_{f}}=\|\nabla \mathbf{u}\|_{0}=\sqrt{(\nabla \mathbf{u}, \nabla \mathbf{u})} & \forall \mathbf{u} \in H_{f}, \\
\|\varphi\|_{H_{p}}=\|\nabla \varphi\|_{0}=\sqrt{(\nabla \varphi, \nabla \varphi)} & \forall \varphi \in H_{p} .
\end{aligned}
$$


We equip the space $W$ with the following norms: $\forall u=(\mathbf{u}, \varphi) \in W$,

$$
\begin{aligned}
& \|u\|_{0}=\sqrt{n(\mathbf{u}, \mathbf{u})_{\Omega_{f}}+\rho g S_{0}(\varphi, \varphi)_{\Omega_{p}}}, \\
& \|u\|_{W}=\sqrt{n \nu(\nabla \mathbf{u}, \nabla \mathbf{u})_{\Omega_{f}}+\rho g K(\nabla \varphi, \nabla \varphi)_{\Omega_{p}}} \approx\|\nabla u\|_{0},
\end{aligned}
$$

where $(\cdot, \cdot)_{D}$ refers to the scalar product $(\cdot, \cdot)$ in the corresponding domain $D$ for $D=\Omega_{f}$ or $\Omega_{p}$, and $\approx$ refers to equivalent norms.

For simplicity, we assume that $n, \rho, g, S_{0}, \nu$ and $K$ are constants. The given data $\mathbf{u}_{f}^{0}, \varphi^{0}, \mathbf{g}_{f}$, and $g_{p}$ are assumed to be smooth enough. The weak formulation of the above non-stationary Stokes-Darcy model reads as follows: find $u=\left(\mathbf{u}_{f}, \varphi\right) \in W$ and $p_{f} \in Q$, such that $\forall t \in(0, T]$,

$$
\begin{aligned}
& \left(\frac{\partial u}{\partial t}, v\right)+a(u, v)+b\left(v, p_{f}\right)=f(v) \forall v=(\mathbf{v}, \psi) \in W, \\
& b(u, q)=0 \forall q \in Q, \\
& u(\mathbf{x}, 0)=u^{0},
\end{aligned}
$$

where

$$
\begin{aligned}
& a(u, v)=a_{\Omega}(u, v)+a_{\Gamma}(u, v), \\
& a_{\Omega}(u, v)=a_{\Omega_{f}}\left(\mathbf{u}_{f}, \mathbf{v}\right)+a_{\Omega_{p}}(\varphi, \psi), \\
& a_{\Omega_{f}}\left(\mathbf{u}_{f}, \mathbf{v}\right)=n \int_{\Omega_{f}} \nu \nabla \mathbf{u}_{f}: \nabla \mathbf{v}+n \sum_{i=1}^{d-1} \int_{\Gamma} \frac{\alpha}{\sqrt{\boldsymbol{\tau}_{i} \cdot \mathbf{K} \boldsymbol{\tau}_{i}}}\left(\mathbf{u}_{f} \cdot \boldsymbol{\tau}_{i}\right)\left(\mathbf{v} \cdot \boldsymbol{\tau}_{i}\right), \\
& a_{\Omega_{p}}(\varphi, \psi)=\rho g \int_{\Omega_{p}} \mathbf{K} \nabla \varphi \cdot \nabla \psi, \\
& a_{\Gamma}(u, v)=n \rho g \int_{\Gamma}\left(\varphi \mathbf{v} \cdot \mathbf{n}_{f}-\psi \mathbf{u}_{f} \cdot \mathbf{n}_{f}\right), \\
& b\left(v, p_{f}\right)=-n \int_{\Omega_{f}} p_{f} \operatorname{div} \mathbf{v}, \\
& f(v)=n \int_{\Omega_{f}} \mathbf{g}_{f} \cdot \mathbf{v}+\rho g \int_{\Omega_{p}} g_{p} \psi .
\end{aligned}
$$

The well-posedness of the mixed Stokes-Darcy model (1.16)-(1.18) can be found in [6, 7] for the stationary case and is assumed to hold similarly for the non-stationary case. In this paper, we focus on its numerical solution.

Throughout this paper, we will use letters $C$ and $\tilde{C}$ (with or without subscripts) to denote generic positive constants independent of the discretization parameters $k$ and $h$. For convenience, we will use the notation $x \lesssim y$ to denote that $x \leq C y$. We will also denote $u(t)=\left(\mathbf{u}_{f}(t), \varphi(t)\right), p_{f}(t)$, etc., by omitting $\mathbf{x}$ when there is no confusion.

It is known [6] that $a_{\Omega_{f}}(\cdot, \cdot), a_{\Omega_{p}}(\cdot, \cdot)$, and $a_{\Gamma}(\cdot, \cdot)$ are continuous, and $a(\cdot, \cdot)$ is coercive. Namely,

$$
\begin{gathered}
a_{\Omega_{f}}(\mathbf{u}, \mathbf{v}) \leq C\|\mathbf{u}\|_{H_{f}}\|\mathbf{v}\|_{H_{f}} \forall \mathbf{u}, \mathbf{v} \in H_{f}, \\
a_{\Omega_{p}}(\phi, \psi) \leq C\|\phi\|_{H_{p}}\|\psi\|_{H_{p}} \forall \phi, \psi \in H_{p}, \\
a_{\Gamma}(u, v) \leq C\|u\|_{W}\|v\|_{W} \forall u, v \in W, \\
a(v, v) \geq \alpha_{0}\|v\|_{W}^{2} \forall v \in W,
\end{gathered}
$$


where $\alpha_{0}$ is a positive constant. Furthermore, $a_{\Omega_{f}}(\cdot, \cdot)$ and $a_{\Omega_{p}}(\cdot, \cdot)$ are symmetric,

$$
a_{\Gamma}(u, v)=-a_{\Gamma}(v, u) \text { and } a_{\Gamma}(u, u)=0 \quad \forall u, v \in W .
$$

We are interested in decoupled numerical methods for the mixed model. There are many appealing reasons as discussed in [18, that have led to active research on developing effective and efficient decoupling techniques for multi-physics mixed models so that existing single-model solvers can be applied locally with little extra computational and software overhead. Most of the decoupled methods for the Stokes-Darcy model in the literature are developed for the stationary case. For example, iterative subdomain methods based on domain decomposition are proposed and studied in 6, 7, 8, 12, a Lagrange multiplier based approach is suggested in 15]; a two-grid method is proposed in [18; and several decoupled pre-conditioners are constructed in [5]. For the non-stationary case, a Dirichlet-Neumann iterative method is proposed and numerical experiments are reported in [6. In this paper, a decoupling approach based on interface approximation via temporal extrapolation is proposed for devising decoupled marching algorithms for the mixed model so that at each time level, two decoupled subproblems are solved independently by invoking a Stokes solver and a Darcy solver, respectively. Error estimates are derived and numerical experiments are conducted to demonstrate the computational effectiveness of the decoupling approach.

The rest of the paper is organized as follows. Both coupled and decoupled marching algorithms are presented in Section 2. The error analysis is given in Section 3. Numerical experiments are reported in Section 4 , followed by conclusions in Section 5 ,

\section{Numerical ALGORIthmS}

We consider a triangulation $\mathcal{T}_{h}$ of the domain $\bar{\Omega}_{f} \cup \bar{\Omega}_{p}$, depending on a positive parameter $h>0$, made up of triangles if $d=2$, or tetrahedra if $d=3$. We assume as in [6] that:

(1) each triangle or tetrahedra, say $T$, is such that $\operatorname{int}(T) \neq \emptyset$;

(2) $\operatorname{int}\left(T_{1}\right) \cap \operatorname{int}\left(T_{2}\right)=\emptyset$ for each pair of different $T_{1}, T_{2} \in \mathcal{T}_{h}$, and if $T_{1} \cap T_{2}=$ $F \neq \emptyset$, then $F$ is a common face or edge or vertex to $T_{1}$ and $T_{2}$;

(3) $\operatorname{diam}(T) \leq h$ for all $T \in \mathcal{T}_{h}$;

(4) $\mathcal{T}_{h}$ is regular; that is, there exists a constant $C_{r} \geq 1$ such that

$$
\max _{T \in \mathcal{T}_{h}} \frac{\operatorname{diam}(T)}{\rho_{T}} \leq C_{r} \forall h>0
$$

with $\rho_{T}=\sup \{\operatorname{diam}(B): B$ is a ball contained in $T\}$;

(5) the triangulations $\mathcal{T}_{f h}$ and $\mathcal{T}_{p h}$ induced on the subdomains $\Omega_{f}$ and $\Omega_{p}$ are compatible on the interface $\Gamma$; that is, they share the same edges (if $d=2$ ) or faces (if $d=3$ ) therein;

(6) the triangulation $\mathcal{T}_{\Gamma h}$ induced on $\Gamma$ is quasi-uniform; that is, it is regular and there exists a constant $C_{\Gamma}>0$ such that $\min _{T \in \mathcal{T}_{\Gamma h}} h_{T} \geq C_{\Gamma} h$ for all $h>0$.

Let $W_{h}=H_{f h} \times H_{p h} \subset W$ and $Q_{h} \subset Q$ denote the finite element subspaces as introduced for the stationary mixed model in [6, 18. The finite element spaces $H_{f h}$ and $Q_{h}$ approximating velocity and pressure in the fluid flow region are assumed to 
satisfy the well known discrete inf-sup condition: There exists a positive constant $\beta$, independent of $h$, such that $\forall q_{h} \in Q_{h}, \exists v_{h} \in W_{h}, v_{h} \neq 0$,

$$
b\left(v_{h}, q_{h}\right) \geq \beta\left\|v_{h}\right\|_{W}\left\|q_{h}\right\|_{Q} .
$$

Several families of finite element spaces designed for the Stokes problem are provided in 4. They all satisfy the discrete inf-sup condition (2.1) and can thus be applied for $H_{f h}$ and $Q_{h}$. Standard finite element approximations of $H^{1}\left(\Omega_{p}\right)$ can be applied for $H_{p h}$ in the porous media flow region. For illustration, we assume that the finite element spaces of the first-order approximation $O(h)$ are used in the fluid flow, such as the well-known MINI elements, and the porous media flow regions, such as the linear Lagrangian elements. The corresponding inverse estimates are well known:

$$
\begin{aligned}
\left\|\nabla \mathbf{v}_{h}\right\|_{0} \leq C h^{-1}\left\|\mathbf{v}_{h}\right\|_{0} \quad & \forall \mathbf{v}_{h} \in H_{f h}, \\
\left\|\nabla \psi_{h}\right\|_{0} \leq C h^{-1}\left\|\psi_{h}\right\|_{0} & \forall \psi_{h} \in H_{p h} .
\end{aligned}
$$

The following estimates on the coupling term are also useful in our analysis.

Lemma 1. $\forall u=(\mathbf{u}, \phi), v=(\mathbf{v}, \psi) \in W$, there exists $C>0$ such that $\forall \varepsilon>0$,

$$
\left|a_{\Gamma}(u, v)\right| \leq \frac{1}{4 \varepsilon}\|u\|_{W}^{2}+C \varepsilon\|v\|_{W}^{2} .
$$

In addition, $\forall u_{h}=\left(\mathbf{u}_{h}, \phi_{h}\right), v_{h}=\left(\mathbf{v}_{h}, \psi_{h}\right) \in W_{h}$, there exists $C>0$ such that

$$
\left|a_{\Gamma}\left(u_{h}, v_{h}\right)\right| \leq \frac{1}{4 \varepsilon}\left\|u_{h}\right\|_{W}^{2}+C \varepsilon h^{-1}\left\|v_{h}\right\|_{0}^{2} .
$$

Proof. (2.4) is a trivial extension of (1.19c).

Furthermore, from a refined trace estimate (see [24], page 17, with $\epsilon=h^{1 / 2}$ ) and the inverse inequalities (2.2)-(2.3),

$$
\begin{aligned}
\left|a_{\Gamma}\left(u_{h}, v_{h}\right)\right| \leq \tilde{C}\left[\left\|\nabla \phi_{h}\right\|_{0}\left(h^{-1 / 2}\left\|\mathbf{v}_{h}\right\|_{0}+h^{1 / 2}\left\|\nabla \mathbf{v}_{h}\right\|_{0}\right)\right. \\
\left.\quad+\left\|\nabla \mathbf{u}_{h}\right\|_{0}\left(h^{-1 / 2}\left\|\psi_{h}\right\|_{0}+h^{1 / 2}\left\|\nabla \psi_{h}\right\|_{0}\right)\right] \\
\leq \tilde{C} h^{-1 / 2}\left(\left\|\nabla \phi_{h}\right\|_{0}\left\|\mathbf{v}_{h}\right\|_{0}+\left\|\nabla \mathbf{u}_{h}\right\|_{0}\left\|\psi_{h}\right\|_{0}\right) \\
\leq\left(\frac{1}{4 \varepsilon} \rho g K\left\|\nabla \phi_{h}\right\|_{0}^{2}+C \varepsilon h^{-1}\left\|\mathbf{v}_{h}\right\|_{0}^{2}\right)+\left(\frac{1}{4 \varepsilon} n \nu\left\|\nabla \mathbf{u}_{h}\right\|_{0}^{2}+C \varepsilon h^{-1}\left\|\psi_{h}\right\|_{0}^{2}\right) \\
\leq \frac{1}{4 \varepsilon}\left\|u_{h}\right\|_{W}^{2}+C \varepsilon h^{-1}\left\|v_{h}\right\|_{0}^{2},
\end{aligned}
$$

which proves (2.5).

We also introduce a subspace $V_{h}$ of $W_{h}$ defined by

$$
V_{h}=\left\{v_{h} \in W_{h}: b\left(v_{h}, q_{h}\right)=0 \forall q_{h} \in Q_{h}\right\},
$$

and correspondingly, the projection $R_{h}: v=(\mathbf{v}, \psi) \in W \mapsto R_{h} v=\left(R_{h} \mathbf{v}, R_{h} \psi\right) \in$ $V_{h}$ defined by

$$
\left(\left(R_{h} v, v_{h}\right)\right)=\left(\left(v, v_{h}\right)\right) \quad v \in W, v_{h} \in V_{h},
$$

where

$$
((u, v))=n \nu(\nabla \mathbf{u}, \nabla \mathbf{v})_{\Omega_{f}}+\rho g K(\nabla \phi, \nabla \psi)_{\Omega_{p}} \forall u=(\mathbf{u}, \phi), v=(\mathbf{v}, \psi) \in W .
$$

If $v \in W \cap\left\{\left(H^{2}\left(\Omega_{f}\right)\right)^{d} \times H^{2}\left(\Omega_{p}\right)\right\}$, we have the error estimates:

$$
\left\|R_{h} v-v\right\|_{0} \lesssim h^{2},\left\|\nabla\left(R_{h} v-v\right)\right\|_{0} \lesssim h .
$$


Without loss of generality, we assume a uniform mesh applied to the time interval $[0, T]$ with $t_{m}=m k, m=0,1, \ldots, J$, where $k=\frac{T}{J}$ is the time step.

2.1. Coupled marching schemes for the mixed model. Recall that the mixed model (1.16)-1.17) is formulated as an abstract time-dependent saddle-point problem. It is natural to consider the following first-order implicit marching scheme by applying the backward divided difference for the temporal discretization and the finite element Galerkin method for the spacial discretization, which leads to the coupled backward Euler scheme:

Algorithm 1. Coupled Backward Euler Scheme (CBES)

Find $u_{h}^{m}=\left(\mathbf{u}_{f h}^{m}, \varphi_{h}^{m}\right) \in W_{h}$ and $p_{f h}^{m} \in Q_{h}, m=1, \ldots, J$, such that $\forall v_{h}=$ $\left(\mathbf{v}_{h}, \psi_{h}\right) \in W_{h}$ and $\forall q_{h} \in Q_{h}$,

$$
\begin{aligned}
& \left(\frac{u_{h}^{m}-u_{h}^{m-1}}{k}, v_{h}\right)+a\left(u_{h}^{m}, v_{h}\right)+b\left(v_{h}, p_{f h}^{m}\right)=f^{m}\left(v_{h}\right), \\
& b\left(u_{h}^{m}, q_{h}\right)=0 \\
& u_{h}^{0}=R_{h} u^{0},
\end{aligned}
$$

where $f^{m}=f\left(t_{m}\right), u^{0}=\left(\mathbf{u}_{f}^{0}, \varphi^{0}\right)$.

Note that at each time level, CBES amounts to solving a stationary mixed Stokes-Darcy problem and is thus well-posed. Theoretical analysis and numerical experiments in the following sections will show that CBES is unconditionally stable and error estimates are also given. Higher-order marching schemes, such as the Crank-Nicolson scheme, may be applied if necessary. As in the stationary case, the major concern here is that a coupled problem must be solved at each time level. In principle, the decoupled methods developed for the stationary model can all be applied here at each time level. However, those methods either involve an iterative procedure, or a coarse-grid solver, or have other overhead and disadvantages.

2.2. Decoupled marching schemes for the mixed model. We now propose a decoupling approach based on the temporal extrapolation on the interface, which will lead to efficient decoupled marching algorithms and easy implementation. Recall that $a(\cdot, \cdot)=a_{\Omega}(\cdot, \cdot)+a_{\Gamma}(\cdot, \cdot)$. The key idea of the two-grid decoupling approach for the stationary model in [18] is to approximate the interface coupling term $a_{\Gamma}(\cdot, \cdot)$ by a coarse-grid solution. A straightforward extension of the two-grid approach to the time-dependent setting is to approximate the term $a_{\Gamma}\left(u_{h}^{m}, v_{h}\right)$ in the coupled algorithm CBES by $a_{\Gamma}\left(u_{H}^{m}, v_{h}\right)$, which, however, requires solving a coupled coarsegrid problem for $u_{H}^{m}$ at each time step. It is important to note that the coarse-grid approximation may, in principle, be replaced by any other approximation with comparable accuracy. Thanks to the nature of time-dependent problems that the computed solutions from previous time steps can provide useful information for various approximation purposes via suitable temporal extrapolation techniques, see e.g. [17, we now describe how to approximate $u_{h}^{m}$ in the interface coupling term $a_{\Gamma}\left(u_{h}^{m}, v_{h}\right)$ by an appropriate extrapolation of the computed solutions from the previous time steps. For the backward Euler scheme, for instance, it suffices to approximate $\left.u_{h}^{m}\right|_{\Gamma}$ by the corresponding first-order temporal extrapolation, simply $\left.u_{h}^{m-1}\right|_{\Gamma}$, which leads to the following decoupled backward Euler scheme:

Algorithm 2. Decoupled Backward Euler Scheme (DBES)

Find $u^{h, m}=\left(\mathbf{u}_{f}^{h, m}, \varphi^{h, m}\right) \in W_{h}$ and $p_{f}^{h, m} \in Q_{h}, m=1, \ldots, J$, such that $\forall v_{h}=$ 
$\left(\mathbf{v}_{h}, \psi_{h}\right) \in W_{h}$ and $\forall q_{h} \in Q_{h}$,

$$
\begin{aligned}
& \left(\frac{u^{h, m}-u^{h, m-1}}{k}, v_{h}\right)+a_{\Omega}\left(u^{h, m}, v_{h}\right)+b\left(v_{h}, p_{f}^{h, m}\right) \\
& \quad=f^{m}\left(v_{h}\right)-a_{\Gamma}\left(u^{h, m-1}, v_{h}\right), \\
& b\left(u^{h, m}, q_{h}\right)=0, \\
& u^{h, 0}=R_{h} u^{0} .
\end{aligned}
$$

It is easy to see that DBES is also well-posed. Furthermore, at each time step, the discrete model (2.13)-(2.15) is equivalent to two decoupled problems that correspond to a Stokes problem in $\Omega_{f}$ and a Darcy problem in $\Omega_{p}$, respectively, with associated boundary conditions defined by $u^{h, m-1}$ from the previous time level on $\Gamma$. More specifically, the discrete Stokes problem in the fluid region $\Omega_{f}$ reads as follows: Find $\mathbf{u}_{f}^{h, m} \in H_{f h}$ and $p_{f}^{h, m} \in Q_{h}, m=1, \ldots, J$, such that $\forall \mathbf{v}_{h} \in H_{f h}$ and $\forall q_{h} \in Q_{h}$,

$$
\begin{aligned}
& n\left(\frac{\mathbf{u}_{f}^{h, m}-\mathbf{u}_{f}^{h, m-1}}{k}, \mathbf{v}_{h}\right)+a_{\Omega_{f}}\left(\mathbf{u}_{f}^{h, m}, \mathbf{v}_{h}\right)+b_{\Omega_{f}}\left(\mathbf{v}_{h}, p_{f}^{h, m}\right) \\
& \quad=n \int_{\Omega_{f}} \mathbf{g}_{f}^{m} \cdot \mathbf{v}_{h}-n \rho g \int_{\Gamma} \varphi^{h, m-1} \mathbf{v}_{h} \cdot \mathbf{n}_{f}, \\
& b_{\Omega_{f}}\left(\mathbf{u}_{f}^{h, m}, q_{h}\right)=0, \\
& \mathbf{u}_{f}^{h, 0}=R_{h} \mathbf{u}_{f}^{0},
\end{aligned}
$$

where $b_{\Omega_{f}}\left(\mathbf{v}_{h}, q_{h}\right)=-n \int_{\Omega_{f}} q_{h} \operatorname{divv}_{h}$, and the discrete Darcy problem in the porous media region $\Omega_{p}$ reads as follows: Find $\varphi^{h, m} \in H_{p h}, m=1, \ldots, J$, such that $\forall \psi_{h} \in H_{p h}$,

$\rho g S_{0}\left(\frac{\varphi^{h, m}-\varphi^{h, m-1}}{k}, \psi_{h}\right)+a_{\Omega_{p}}\left(\varphi^{h, m}, \psi_{h}\right)=\rho g \int_{\Omega_{p}} g_{p}^{m} \psi_{h}+n \rho g \int_{\Gamma} \psi_{h} \mathbf{u}_{f}^{h, m-1} \cdot \mathbf{n}_{f}$, $\varphi^{h, 0}=R_{h} \varphi^{0}$.

Similarly, higher-order extrapolations may applied to further improve the approximation accuracy, if necessary. For instance, one may approximate $u_{h}^{m}$ by $2 u_{h}^{m-1}-u_{h}^{m-2}$ in the interface coupling term due to the second-order extrapolation

$$
u^{m}=2 u^{m-1}-u^{m-2}+O\left(k^{2}\right) .
$$

\section{ERror ANALYSIS}

The error analysis for the decoupled algorithm DBES is divided into two parts. First, we derive error estimates for the coupled scheme CBES, which is more convenient than directly working on the decoupled scheme. Then, we compare the difference between the coupled and decoupled schemes, which finally leads to the required error estimates for the decoupled scheme.

For convenience, let us introduce the following notation. We denote the backward divided difference operator $d_{t}$ by

$$
d_{t} u_{h}^{m}=\frac{u_{h}^{m}-u_{h}^{m-1}}{k}, \text { for } m=1, \ldots, J .
$$


When $m=0$, we define $d_{t} u_{h}^{0}=\left(d_{t} \mathbf{u}_{f h}^{0}, d_{t} \varphi_{h}^{0}\right)$ as the solution to the following problem:

$$
\left(d_{t} u_{h}^{0}, v_{h}\right)+a\left(u_{h}^{0}, v_{h}\right)=f^{0}\left(v_{h}\right) \quad \forall v_{h} \in V_{h}
$$

We also denote

$$
d_{t t} u_{h}^{m}=\frac{d_{t} u_{h}^{m}-d_{t} u_{h}^{m-1}}{k}, \text { for } m=1, \ldots, J
$$

Similarly, we also introduce the divided differences $d_{t} u^{h, m}$ and $d_{t t} u^{h, m}$, for $m=$ $1, \ldots, J$, for the solution $u^{h, m}$ of DBES, and for $m=0, d_{t} u^{h, 0}$ is defined in the same way as $d_{t} u_{h}^{0}$ in (3.2).

It is easy to verify that both $\left\|d_{t} u_{h}^{0}\right\|_{0}$ and $\left\|d_{t} u^{h, 0}\right\|_{0}$ can be bounded from above by constants depending on the given data $u^{0}$ and $f^{0}$.

3.1. Error estimates for CBES. Let us define a projection operator $P_{h}:(w(t)$, $p(t)) \in W \times Q \mapsto\left(P_{h} w(t), P_{h} p(t)\right) \in W_{h} \times Q_{h}, \forall t \in[0, T]$ by

$$
a\left(P_{h} w(t), v_{h}\right)+b\left(v_{h}, P_{h} p(t)\right)=a\left(w(t), v_{h}\right)+b\left(v_{h}, p(t)\right) \quad \forall v_{h} \in W_{h},
$$

Note that (3.4)-(3.5) correspond to a stationary mixed Stokes-Darcy problem and is thus well defined from [6, 7]. Apparently, $P_{h}$ is a linear operator. Therefore, for any $(w(t), p(t)) \in W \times Q$ and $(v(t), q(t)) \in W \times Q$,

$$
\begin{aligned}
& P_{h}(u(t)-v(t))=P_{h} u(t)-P_{h} v(t), \\
& P_{h}(p(t)-q(t))=P_{h} p(t)-P_{h} q(t) .
\end{aligned}
$$

Furthermore, under a certain smoothness assumption on $(w(t), p(t))$, we have the following error estimates:

$$
\begin{aligned}
& \left\|P_{h} w(t)-w(t)\right\|_{W} \lesssim h, \\
& \left\|P_{h} p(t)-p(t)\right\|_{0} \lesssim h
\end{aligned}
$$

from [6] and

$$
\left\|P_{h} w(t)-w(t)\right\|_{0} \lesssim h^{2}
$$

from $[18$.

From now on, we assume that $u(t) \in\left(H^{2}\left(\Omega_{f}\right)\right)^{d} \times H^{2}\left(\Omega_{p}\right), u_{t}(t)$ and $u_{t t}(t) \in$ $\left(L^{2}\left(\Omega_{f}\right)\right)^{d} \times L^{2}\left(\Omega_{p}\right)$ for the solution of the continuous coupled problem (1.16)-(1.18).

Theorem 1. For CBES (2.10)-(2.12), it follows, for $m=1, \ldots, J$, that

$$
\begin{aligned}
& \left\|u_{h}^{m}-u\left(t_{m}\right)\right\|_{0} \lesssim k+h^{2}, \\
& \left\|\nabla\left(u_{h}^{m}-u\left(t_{m}\right)\right)\right\|_{0} \lesssim k+h, \\
& \left\|p_{f h}^{m}-p_{f}\left(t_{m}\right)\right\|_{0} \lesssim k+h+k^{-1} h^{2} .
\end{aligned}
$$

Proof. Observe that

$$
\begin{aligned}
& u_{h}^{m}-u\left(t_{m}\right)=\left[u_{h}^{m}-P_{h} u\left(t_{m}\right)\right]+\left[P_{h} u\left(t_{m}\right)-u\left(t_{m}\right)\right]=\theta_{1}^{m}+\theta_{2}^{m}, \\
& p_{f h}^{m}-p_{f}\left(t_{m}\right)=\left[p_{f h}^{m}-P_{h} p_{f}\left(t_{m}\right)\right]+\left[P_{h} p_{f}\left(t_{m}\right)-p_{f}\left(t_{m}\right)\right]=\zeta_{1}^{m}+\zeta_{2}^{m} .
\end{aligned}
$$

From (3.8), we first have

$$
\left\|\theta_{2}^{m}\right\|_{0} \lesssim h^{2}
$$


In order to estimate $\theta_{1}^{m}$, it is easy to verify that for $m=1, \ldots, J$,

$$
\left(\frac{\theta_{1}^{m}-\theta_{1}^{m-1}}{k}, v_{h}\right)+a\left(\theta_{1}^{m}, v_{h}\right)+b\left(v_{h}, \zeta_{1}^{m}\right)=-\left(\omega^{m}, v_{h}\right),
$$

where

$$
\begin{aligned}
\omega^{m} & =\frac{P_{h} u\left(t_{m}\right)-P_{h} u\left(t_{m-1}\right)}{k}-u_{t}\left(t_{m}\right) \\
& =\left[\frac{P_{h} u\left(t_{m}\right)-P_{h} u\left(t_{m-1}\right)}{k}-\frac{u\left(t_{m}\right)-u\left(t_{m-1}\right)}{k}\right]+\left[\frac{u\left(t_{m}\right)-u\left(t_{m-1}\right)}{k}-u_{t}\left(t_{m}\right)\right] \\
& =\omega_{1}^{m}+\omega_{2}^{m} .
\end{aligned}
$$

Taking $v_{h}=\theta_{1}^{m}$ in (3.15) and observing that $b\left(\theta_{1}^{m}, \zeta_{1}^{m}\right)=0$, we have

$$
\left(\frac{\theta_{1}^{m}-\theta_{1}^{m-1}}{k}, \theta_{1}^{m}\right)+a\left(\theta_{1}^{m}, \theta_{1}^{m}\right)=-\left(\omega^{m}, \theta_{1}^{m}\right) .
$$

Since $a(\cdot, \cdot)$ is coercive, it follows that

$$
\left\|\theta_{1}^{m}\right\|_{0}^{2}-\left(\theta_{1}^{m-1}, \theta_{1}^{m}\right) \leq k\left\|\omega^{m}\right\|_{0}\left\|\theta_{1}^{m}\right\|_{0} .
$$

Thus,

$$
\left\|\theta_{1}^{m}\right\|_{0} \leq\left\|\theta_{1}^{m-1}\right\|_{0}+k\left\|\omega^{m}\right\|_{0}
$$

Observe that

$$
\omega_{1}^{j}=\left(P_{h}-I\right) \frac{u\left(t_{j}\right)-u\left(t_{j-1}\right)}{k}=k^{-1} \int_{t_{j-1}}^{t_{j}}\left(P_{h}-I\right) u_{t}(t) d t .
$$

So,

$$
k \sum_{j=1}^{m}\left\|\omega_{1}^{j}\right\|_{0} \leq \sum_{j=1}^{m} \int_{t_{j-1}}^{t_{j}}\left\|\left(P_{h}-I\right) u_{t}(t)\right\|_{0} d t \lesssim h^{2} .
$$

Furthermore,

$$
k \omega_{2}^{j}=u\left(t_{j}\right)-u\left(t_{j-1}\right)-k u_{t}\left(t_{j}\right)=-\int_{t_{j-1}}^{t_{j}}\left(t-t_{j-1}\right) u_{t t}(t) d t .
$$

Thus,

$$
k \sum_{j=1}^{m}\left\|\omega_{2}^{j}\right\|_{0} \leq k \sum_{j=1}^{m} \int_{t_{j-1}}^{t_{j}}\left\|u_{t t}(t)\right\|_{0} d t \lesssim k .
$$

Finally note that $\left\|\theta_{1}^{0}\right\|_{0} \leq\left\|u_{h}^{0}-u(0)\right\|_{0}+\left\|u(0)-P_{h} u(0)\right\|_{0} \lesssim h^{2}$. Similarly, $\left\|\nabla \theta_{1}^{0}\right\|_{0} \lesssim$ $h$. Therefore, from (3.16) we obtain

$$
\left\|\theta_{1}^{m}\right\|_{0} \lesssim k+h^{2} .
$$

Estimate (3.9) then follows from (3.12), (3.14) and (3.20).

Now we prove estimate (3.10). Taking $v_{h}=2\left(\theta_{1}^{m}-\theta_{1}^{m-1}\right)$ in (3.15) and noting that

$$
b\left(2\left(\theta_{1}^{m}-\theta_{1}^{m-1}\right), \zeta_{1}^{m}\right)=0, \text { for } m=1, \ldots, J,
$$

we have

$$
\left(\frac{\theta_{1}^{m}-\theta_{1}^{m-1}}{k}, 2\left(\theta_{1}^{m}-\theta_{1}^{m-1}\right)\right)+a\left(\theta_{1}^{m}, 2\left(\theta_{1}^{m}-\theta_{1}^{m-1}\right)\right)=-\left(\omega^{m}, 2\left(\theta_{1}^{m}-\theta_{1}^{m-1}\right)\right) .
$$


Therefore,

$$
\begin{aligned}
& 2 k\left(d_{t} \theta_{1}^{m}, d_{t} \theta_{1}^{m}\right)+2 a_{\Omega}\left(\theta_{1}^{m}, \theta_{1}^{m}-\theta_{1}^{m-1}\right) \\
& =-2 k\left(\omega^{m}, d_{t} \theta_{1}^{m}\right)-2 a_{\Gamma}\left(\theta_{1}^{m}, \theta_{1}^{m}-\theta_{1}^{m-1}\right) \\
& =-2 k\left(\omega^{m}, d_{t} \theta_{1}^{m}\right)+2 k a_{\Gamma}\left(d_{t} \theta_{1}^{m}, \theta_{1}^{m-1}\right) \\
& \leq k\left\|\omega^{m}\right\|_{0}^{2}+k\left\|d_{t} \theta_{1}^{m}\right\|_{0}^{2}+k^{2}\left\|d_{t} \theta_{1}^{m}\right\|_{W}^{2}+(C n \rho g)^{2}\left\|\theta_{1}^{m-1}\right\|_{W}^{2} .
\end{aligned}
$$

Then we have

$$
\left\|\theta_{1}^{m}\right\|_{W}^{2}-\left\|\theta_{1}^{m-1}\right\|_{W}^{2}+k\left\|d_{t} \theta_{1}^{m}\right\|_{0}^{2} \leq(C n \rho g)^{2}\left\|\theta_{1}^{m-1}\right\|_{W}^{2}+k\left\|\omega^{m}\right\|_{0}^{2},
$$

which leads to

$$
\left\|\theta_{1}^{m}\right\|_{W}^{2}+k \sum_{j=1}^{m}\left\|d_{t} \theta_{1}^{j}\right\|_{0}^{2} \leq \sum_{j=0}^{m-1}(C n \rho g)^{2}\left\|\theta_{1}^{j}\right\|_{W}^{2}+k \sum_{j=1}^{m}\left\|\omega^{j}\right\|_{0}^{2}+\left\|\theta_{1}^{0}\right\|_{W}^{2} .
$$

It follows from Gronwall's lemma that

$$
\left\|\theta_{1}^{m}\right\|_{W}^{2}+k \sum_{j=1}^{m}\left\|d_{t} \theta_{1}^{j}\right\|_{0}^{2} \leq \exp \left(\sum_{j=0}^{m-1}(C n \rho g)^{2}\right)\left(k \sum_{j=1}^{m}\left\|\omega_{1}^{j}\right\|_{0}^{2}+k \sum_{j=1}^{m}\left\|\omega_{2}^{j}\right\|_{0}^{2}+\left\|\nabla \theta_{1}^{0}\right\|_{0}^{2}\right) .
$$

Note that

$$
k \sum_{j=1}^{m}\left\|\omega_{1}^{j}\right\|_{0}^{2} \lesssim h^{4}
$$

and

$$
\begin{aligned}
k \sum_{j=1}^{m}\left\|\omega_{2}^{j}\right\|_{0}^{2} & \leq k^{-1} \sum_{j=1}^{m} k^{2}\left(\int_{t_{j-1}}^{t_{j}}\left\|u_{t t}(t)\right\|_{0} d t\right)^{2} \\
& \leq k \sum_{j=1}^{m}\left[\left(\int_{t_{j-1}}^{t_{j}} 1^{2} d t\right)\left(\int_{t_{j-1}}^{t_{j}}\left\|u_{t t}(t)\right\|_{0}^{2} d t\right)\right]=k^{2} \int_{0}^{t_{m}}\left\|u_{t t}(t)\right\|_{0}^{2} d t .
\end{aligned}
$$

So,

$$
\left\|\theta_{1}^{m}\right\|_{W}^{2}+\sum_{j=1}^{m} k\left\|d_{t} \theta_{1}^{j}\right\|_{0}^{2} \lesssim h^{4}+k^{2} \int_{0}^{t_{m}}\left\|u_{t t}(s)\right\|_{0} d s+\left\|\nabla \theta_{1}^{0}\right\|_{0}^{2} \lesssim k^{2}+h^{2},
$$

which implies

$$
\left\|\nabla \theta_{1}^{m}\right\|_{0} \lesssim k+h .
$$

Also note that $\left\|\nabla \theta_{2}^{m}\right\|_{0} \lesssim h$ from (3.6). Estimate (3.10) then follows.

Finally, we prove the estimate for pressure (3.11). Note from (3.13) that

$$
\left\|p_{f h}^{m}-p_{f}\left(t_{m}\right)\right\|_{0} \leq\left\|\zeta_{1}^{m}\right\|_{0}+\left\|\zeta_{2}^{m}\right\|_{0} .
$$

First, we have from (3.7),

$$
\left\|\zeta_{2}^{m}\right\|_{0} \lesssim h
$$

To estimate $\left\|\zeta_{1}^{m}\right\|_{0}$, let us start with the estimate for $\left\|d_{t} \theta_{1}^{m}\right\|_{0}$. Subtracting equation (3.15) on the two adjacent time levels, we have for $m=2, \ldots, J$,

$$
\left(\frac{d_{t} \theta_{1}^{m}-d_{t} \theta_{1}^{m-1}}{k}, v_{h}\right)+a\left(d_{t} \theta_{1}^{m}, v_{h}\right)+\frac{1}{k} b\left(v_{h}, \zeta_{1}^{m}-\zeta_{1}^{m-1}\right)=-\left(d_{t} \omega^{m}, v_{h}\right)
$$


Taking $v_{h}=d_{t} \theta_{1}^{m}$ in (3.28) and observing that $b\left(d_{t} \theta_{1}^{m}, \zeta_{1}^{m}-\zeta_{1}^{m-1}\right)=0$, then similarly to (3.16), we obtain

$$
\left\|d_{t} \theta_{1}^{m}\right\|_{0} \leq\left\|d_{t} \theta_{1}^{1}\right\|_{0}+k \sum_{j=2}^{m}\left\|d_{t} \omega^{j}\right\|_{0} \leq\left\|d_{t} \theta_{1}^{1}\right\|_{0}+k \sum_{j=2}^{m}\left\|d_{t} \omega_{1}^{j}\right\|_{0}+k \sum_{j=2}^{m}\left\|d_{t} \omega_{2}^{j}\right\|_{0} .
$$

By definition,

$$
\left\|d_{t} \theta_{1}^{1}\right\|_{0}=\frac{1}{k}\left\|\theta_{1}^{1}-\theta_{1}^{0}\right\|_{0} \leq \frac{1}{k}\left(\left\|\theta_{1}^{1}\right\|_{0}+\left\|\theta_{1}^{0}\right\|_{0}\right) .
$$

Recall that $\left\|\theta_{1}^{0}\right\|_{0} \lesssim h^{2}$, and we also have

$$
\left\|\theta_{1}^{1}\right\|_{0} \lesssim k^{2}+h^{2}
$$

from (3.19) and (3.16). It follows that

$$
\left\|d_{t} \theta_{1}^{1}\right\|_{0} \lesssim k+k^{-1} h^{2} .
$$

On the other hand, we have from (3.17),

$$
d_{t} \omega_{1}^{j}=\frac{\omega_{1}^{j}-\omega_{1}^{j-1}}{k}=\left(P_{h}-I\right) \frac{u\left(t_{j}\right)-2 u\left(t_{j-1}\right)+u\left(t_{j-2}\right)}{k^{2}} .
$$

Thus,

$$
k \sum_{j=2}^{m}\left\|d_{t} \omega_{1}^{j}\right\|_{0}=k \sum_{j=2}^{m} \max _{t_{j-2} \leq t \leq t_{j}}\left\|\left(P_{h}-I\right) u_{t t}(t)\right\|_{0} \lesssim h^{2} .
$$

Furthermore,

$$
d_{t} \omega_{2}^{j}=\frac{\omega_{2}^{j}-\omega_{2}^{j-1}}{k}=-\frac{1}{2}\left[u_{t t}\left(s_{j 0}\right)-u_{t t}\left(s_{j 1}\right)\right],
$$

for some $s_{j 0} \in\left(t_{j-1}, t_{j}\right), s_{j 1} \in\left(t_{j-2}, t_{j-1}\right)$. Then

$$
k \sum_{j=2}^{m}\left\|d_{t} \omega_{2}^{j}\right\|_{0} \leq \frac{1}{2} k^{2} J \max _{0 \leq t \leq T}\left\|u_{t t t}(t)\right\|_{0} \lesssim k .
$$

Combining (3.29) with (3.30), (3.31) and (3.32), we arrive at

$$
\left\|d_{t} \theta_{1}^{m}\right\|_{0} \lesssim k+k^{-1} h^{2}, \text { for } m=1, \ldots, J .
$$

In summary, we have from (3.15),

$$
b\left(v_{h}, \zeta_{1}^{m}\right) \leq C\left\|\nabla v_{h}\right\|_{0}\left(\left\|d_{t} \theta_{1}^{m}\right\|_{0}+\left\|\nabla \theta_{1}^{m}\right\|_{0}+\left\|\omega^{m}\right\|_{0}\right) .
$$

Therefore, from the discrete inf-sup condition (2.1), it follows that

$$
\left\|\zeta_{1}^{m}\right\|_{0} \leq C \beta^{-1}\left(\left\|d_{t} \theta_{1}^{m}\right\|_{0}+\left\|\nabla \theta_{1}^{m}\right\|_{0}+\left\|\omega^{m}\right\|_{0}\right) \lesssim k+h+k^{-1} h^{2},
$$

by using (3.25), (3.33), as well as the following estimate for $\left\|\omega^{m}\right\|_{0}$ :

$$
\begin{aligned}
\left\|\omega^{m}\right\|_{0} \leq\left\|\omega_{1}^{m}\right\|_{0}+\left\|\omega_{2}^{m}\right\|_{0} & \leq k^{-1} \int_{t_{m-1}}^{t_{m}}\left\|\left(P_{h}-I\right) u_{t}(t)\right\|_{0} d t+C k \\
& \lesssim k^{-1}\left(k h^{2}\right)+C k \lesssim k+h^{2} .
\end{aligned}
$$

Thus, (3.11) follows from (3.26), (3.27) and (3.34). This completes the proof of the theorem. 
3.2. Error estimates for DBES. We now turn to the error estimates for DBES (2.13)-(2.15). For this purpose, let us compare the difference $\left(e^{m}, \eta^{m}\right)$ between the solution of the decoupled scheme (2.13)-(2.15) and the solution of the coupled scheme (2.10)-(2.12) due to the interface decoupling by temporal approximation, where $\eta^{m}=p_{f h}^{m}-p_{f}^{h, m}$ and $e^{m}=\left(\mathbf{e}^{m}, \xi^{m}\right)$ with $\mathbf{e}^{m}=\mathbf{u}_{f h}^{m}-\mathbf{u}_{f}^{h, m}$ and $\xi^{m}=$ $\varphi_{h}^{m}-\varphi^{h, m}$. In particular, $e^{0}=(\mathbf{0}, 0)$.

Observing the difference between the coupled scheme (2.10)-(2.11) and the decoupled scheme (2.13)-(2.14), we have the following governing equations for $\left(e^{m}, \eta^{m}\right)$, $m=1, \ldots, J$ :

$$
\begin{gathered}
\left(\frac{e^{m}-e^{m-1}}{k}, v_{h}\right)+a_{\Omega}\left(e^{m}, v_{h}\right)+a_{\Gamma}\left(u_{h}^{m}-u^{h, m-1}, v_{h}\right)+b\left(v_{h}, \eta^{m}\right)=0 \forall v_{h} \in W_{h}, \\
(3.36) \quad b\left(e^{m}, q_{h}\right)=0 \forall q_{h} \in Q_{h} .
\end{gathered}
$$

The following lemma gives upper bounds for the solution $u_{h}^{m}$ of CBES, which will be used in the error estimates for $e^{m}$ in Theorem 2, For completeness, the proof of Lemma 2 is included in the Appendix.

Lemma 2. For CBES (2.10)-(2.12), we have

$$
\left\|d_{t} u_{h}^{J}\right\|_{0}^{2}+k^{2} \sum_{m=1}^{J}\left\|d_{t t} u_{h}^{m}\right\|_{0}^{2}+k \sum_{m=1}^{J}\left\|\nabla d_{t} u_{h}^{m}\right\|_{0}^{2} \leq M_{0} .
$$

Furthermore, there exist constants $C_{1}>0$ and $C_{2}>0$ independent of $h$, such that if

$$
C_{1} h \leq k \leq C_{2} h,
$$

then

$$
\left\|\nabla d_{t} u_{h}^{J}\right\|_{0}^{2}+k \sum_{m=2}^{J}\left\|d_{t t} u_{h}^{m}\right\|_{0}^{2} \leq M_{1} .
$$

The positive constants $M_{0}$ and $M_{1}$ above are independent of $k$ and $h$.

Theorem 2. Under the assumption (3.38), we have for $m=1, \ldots, J$,

$$
\begin{aligned}
& \left\|e^{m}\right\|_{0}^{2}+k \sum_{j=1}^{m}\left\|\nabla e^{j}\right\|_{0}^{2} \lesssim k^{2}, \\
& \left\|\nabla e^{m}\right\|_{0}^{2}+2 k \sum_{j=1}^{m}\left\|d_{t} e^{j}\right\|_{0}^{2} \lesssim k \\
& \left\|\eta^{m}\right\|_{0}^{2} \lesssim k
\end{aligned}
$$


Proof. Taking $v_{h}=2 k e^{m}$ in (3.35), we have

$$
\begin{aligned}
& \left(\frac{e^{m}-e^{m-1}}{k}, 2 k e^{m}\right)+a_{\Omega}\left(e^{m}, 2 k e^{m}\right) \\
& =-a_{\Gamma}\left(u_{h}^{m}-u^{h, m-1}, 2 k e^{m}\right) \\
& =a_{\Gamma}\left(2 k e^{m}, u_{h}^{m}-u_{h}^{m-1}+u_{h}^{m-1}-u^{h, m-1}\right) \\
& =a_{\Gamma}\left(2 k e^{m}, e^{m-1}+k d_{t} u_{h}^{m}\right) \\
& =2 k a_{\Gamma}\left(e^{m}-e^{m-1}, e^{m-1}\right)+2 k^{2} a_{\Gamma}\left(e^{m}, d_{t} u_{h}^{m}\right) \\
& =2 k^{2}\left[a_{\Gamma}\left(d_{t} e^{m}, e^{m-1}\right)+a_{\Gamma}\left(e^{m}, d_{t} u_{h}^{m}\right)\right] \\
& \leq 2 k^{2}\left[\left(\frac{1}{4 k}\left\|e^{m-1}\right\|_{W}^{2}+\tilde{C} k h^{-1}\left\|d_{t} e^{m}\right\|_{0}^{2}\right)+\left(\frac{1}{4 k}\left\|e^{m}\right\|_{W}^{2}+\tilde{C} k\left\|d_{t} u_{h}^{m}\right\|_{W}^{2}\right)\right] \\
& \leq \frac{1}{2} k\left(\left\|e^{m}\right\|_{W}^{2}+\left\|e^{m-1}\right\|_{W}^{2}\right)+\tilde{C} k^{3} h^{-1}\left\|d_{t} e^{m}\right\|_{0}^{2}+\tilde{C} k^{3}\left\|d_{t} u_{h}^{m}\right\|_{W}^{2} \\
& \leq \frac{1}{2} k\left(\left\|e^{m}\right\|_{W}^{2}+\left\|e^{m-1}\right\|_{W}^{2}\right)+k^{2}\left\|d_{t} e^{m}\right\|_{0}^{2}+\tilde{C} k^{3}\left\|d_{t} u_{h}^{m}\right\|_{W}^{2},
\end{aligned}
$$

where the divergence-free property, equality (1.20), the definition of $d_{t} u^{h, m}$, and Lemma 1 with $\varepsilon=k$ are used. Note that the left hand side of (3.43) can be rewritten as

$$
\left\|e^{m}\right\|_{0}^{2}-\left\|e^{m-1}\right\|_{0}^{2}+k^{2}\left\|d_{t} e^{m}\right\|_{0}^{2}+2 k\left\|e^{m}\right\|_{W}^{2}+2 n k \sum_{i=1}^{d-1} \int_{\Gamma} \frac{\alpha}{\sqrt{\boldsymbol{\tau}_{i} \cdot \mathbf{K} \boldsymbol{\tau}_{i}}}\left(\mathbf{e}^{m} \cdot \boldsymbol{\tau}_{i}\right)^{2},
$$

by using the identity (see [11])

$$
2(a-b, a)=\|a\|_{0}^{2}-\|b\|_{0}^{2}+\|a-b\|_{0}^{2} \quad \forall a, b \in L^{2}(D) .
$$

It follows that

$$
\left\|e^{m}\right\|_{0}^{2}-\left\|e^{m-1}\right\|_{0}^{2}+k\left\|e^{m}\right\|_{W}^{2}+\frac{1}{2} k\left(\left\|e^{m}\right\|_{W}^{2}-\left\|e^{m-1}\right\|_{W}^{2}\right) \lesssim k^{3}\left\|d_{t} u_{h}^{m}\right\|_{W}^{2} .
$$

Therefore,

$$
\left\|e^{m}\right\|_{0}^{2}+k \sum_{j=1}^{m}\left\|e^{j}\right\|_{W}^{2} \lesssim k^{2}\left(k \sum_{j=1}^{m}\left\|d_{t} u_{h}^{j}\right\|_{W}^{2}\right) .
$$

Estimate (3.40) then follows from the estimate (3.37) of CBES and the equivalence between $\left\|e^{m}\right\|_{W}$ and $\left\|\nabla e^{m}\right\|_{0}$.

Now we prove the estimate (3.41). By taking $v_{h}=2\left(e^{m}-e^{m-1}\right)$ in (3.35) and using the divergence-free property, we have

$$
\begin{aligned}
& \left(\frac{e^{m}-e^{m-1}}{k}, 2\left(e^{m}-e^{m-1}\right)\right)+a_{\Omega}\left(e^{m}, 2\left(e^{m}-e^{m-1}\right)\right) \\
& +a_{\Gamma}\left(u_{h}^{m}-u^{h, m-1}, 2\left(e^{m}-e^{m-1}\right)\right)=0
\end{aligned}
$$


namely,

$$
\begin{aligned}
& 2 k\left(d_{t} e^{m}, d_{t} e^{m}\right)+2 a_{\Omega}\left(e^{m}, e^{m}-e^{m-1}\right) \\
& =-2 k a_{\Gamma}\left(u_{h}^{m}-u^{h, m-1}, d_{t} e^{m}\right) \\
& =-2 k a_{\Gamma}\left(u_{h}^{m}-u_{h}^{m-1}+u_{h}^{m-1}-u^{h, m-1}, d_{t} e^{m}\right) \\
& =-2 k a_{\Gamma}\left(k d_{t} u_{h}^{m}+e^{m-1}, d_{t} e^{m}\right) \\
& =-2 k^{2} a_{\Gamma}\left(d_{t} u_{h}^{m}, d_{t} e^{m}\right)-2 k a_{\Gamma}\left(e^{m-1}, d_{t} e^{m}\right) \\
& \leq 2 k^{2}\left\|d_{t} u_{h}^{m}\right\|_{L^{2}(\Gamma)}\left\|d_{t} e^{m}\right\|_{L^{2}(\Gamma)}+2 k\left\|e^{m-1}\right\|_{L^{2}(\Gamma)}\left\|d_{t} e^{m}\right\|_{L^{2}(\Gamma)} \\
& \leq 2 C k^{2}\left\|\nabla d_{t} u_{h}^{m}\right\|_{0}\left\|\nabla d_{t} e^{m}\right\|_{0}+2 C k\left\|\nabla e^{m-1}\right\|_{0}\left\|\nabla d_{t} e^{m}\right\|_{0} \\
& \leq 2 C^{2} k \cdot k\left\|\nabla d_{t} u_{h}^{m}\right\|_{0}^{2}+k^{2}\left\|\nabla d_{t} e^{m}\right\|_{0}^{2}+2 C^{2}\left\|\nabla e^{m-1}\right\|_{0}^{2} .
\end{aligned}
$$

The left hand side of (3.47) can be written as

$$
\begin{aligned}
& 2 k\left(d_{t} e^{m}, d_{t} e^{m}\right)+2 a_{\Omega}\left(e^{m}, e^{m}-e^{m-1}\right) \\
& =2 k\left(d_{t} e^{m}, d_{t} e^{m}\right)+2 a_{\Omega}\left(e^{m}-e^{m-1}, e^{m}\right) \\
& =2 k\left(d_{t} e^{m}, d_{t} e^{m}\right)+a_{\Omega}\left(e^{m}, e^{m}\right)-a_{\Omega}\left(e^{m-1}, e^{m-1}\right)+k^{2} a_{\Omega}\left(d_{t} e^{m}, d_{t} e^{m}\right) \\
& =2 k\left\|d_{t} e^{m}\right\|_{0}^{2}+\left\|\nabla e^{m}\right\|_{0}^{2}+n \sum_{i=1}^{d-1} \int_{\Gamma} \frac{\alpha}{\sqrt{\boldsymbol{\tau}_{i} \cdot \mathbf{K} \boldsymbol{\tau}_{i}}}\left(\mathbf{e}^{m} \cdot \boldsymbol{\tau}_{i}\right)^{2} \\
& -\left\|\nabla e^{m-1}\right\|_{0}^{2}-n \sum_{i=1}^{d-1} \int_{\Gamma} \frac{\alpha}{\sqrt{\boldsymbol{\tau}_{i} \cdot \mathbf{K} \boldsymbol{\tau}_{i}}}\left(\mathbf{e}^{m-1} \cdot \boldsymbol{\tau}_{i}\right)^{2} \\
& +k^{2}\left\|\nabla d_{t} e^{m}\right\|_{0}^{2}+n k^{2} \sum_{i=1}^{d-1} \int_{\Gamma} \frac{\alpha}{\sqrt{\boldsymbol{\tau}_{i} \cdot \mathbf{K} \boldsymbol{\tau}_{i}}}\left(d_{t} \mathbf{e}^{m} \cdot \boldsymbol{\tau}_{i}\right)^{2} .
\end{aligned}
$$

Therefore,

$$
\begin{aligned}
& \left\|\nabla e^{m}\right\|_{0}^{2}+n \sum_{i=1}^{d-1} \int_{\Gamma} \frac{\alpha}{\sqrt{\boldsymbol{\tau}_{i} \cdot \mathbf{K} \boldsymbol{\tau}_{i}}}\left(\mathbf{e}^{m} \cdot \boldsymbol{\tau}_{i}\right)^{2}-\left\|\nabla e^{m-1}\right\|_{0}^{2} \\
& -n \sum_{i=1}^{d-1} \int_{\Gamma} \frac{\alpha}{\sqrt{\boldsymbol{\tau}_{i} \cdot \mathbf{K} \boldsymbol{\tau}_{i}}}\left(\mathbf{e}^{m-1} \cdot \boldsymbol{\tau}_{i}\right)^{2}+2 k\left\|d_{t} e^{m}\right\|_{0}^{2} \\
& \leq 2 C^{2} k \cdot k\left\|\nabla d_{t} u_{h}^{m}\right\|_{0}^{2}+2 C^{2}\left\|\nabla e^{m-1}\right\|_{0}^{2} .
\end{aligned}
$$

Then we have

$$
\begin{aligned}
& \left\|\nabla e^{m}\right\|_{0}^{2}+n \sum_{i=1}^{d-1} \int_{\Gamma} \frac{\alpha}{\sqrt{\boldsymbol{\tau}_{i} \cdot \mathbf{K} \boldsymbol{\tau}_{i}}}\left(\mathbf{e}^{m} \cdot \boldsymbol{\tau}_{i}\right)^{2}+2 k \sum_{j=1}^{m}\left\|d_{t} e^{j}\right\|_{0}^{2} \\
& \leq \sum_{j=0}^{m-1} C_{1}\left\|\nabla e^{j}\right\|_{0}^{2}+2 C^{2} k \cdot k \sum_{j=1}^{m}\left\|\nabla d_{t} u_{h}^{j}\right\|_{0}^{2} .
\end{aligned}
$$

By using the estimate (3.37), we arrive at

$$
\left\|\nabla e^{m}\right\|_{0}^{2}+2 k \sum_{j=1}^{m}\left\|d_{t} e^{j}\right\|_{0}^{2} \leq \sum_{j=0}^{m-1} C_{1}\left\|\nabla e^{j}\right\|_{0}^{2}+C_{2} k .
$$

Then the estimate (3.41) follows by using Gronwall's lemma. 
To prove (3.42), we need to estimate $d_{t} e^{m}$. Subtracting (3.35)-3.36) on two adjacent time levels, we have for $m=2, \ldots, J$,

$$
\begin{aligned}
& \left(\frac{d_{t} e^{m}-d_{t} e^{m-1}}{k}, v_{h}\right)+a_{\Omega}\left(d_{t} e^{m}, v_{h}\right)+\frac{1}{k}\left[a_{\Gamma}\left(u_{h}^{m}-u^{h, m-1}, v_{h}\right)\right. \\
& \left.-a_{\Gamma}\left(u_{h}^{m-1}-u^{h, m-2}, v_{h}\right)\right]+\frac{1}{k} b\left(v_{h}, \eta^{m}-\eta^{m-1}\right)=0 \forall v_{h} \in W_{h}, \\
& b\left(d_{t} e^{m}, q_{h}\right)=0 \forall q_{h} \in Q_{h} .
\end{aligned}
$$

Taking $v_{h}=2 k d_{t} e^{m}$ in (3.50) and using the divergence-free property and Lemma 1 with $\varepsilon=k$, we obtain

$$
\begin{aligned}
& 2\left(d_{t} e^{m}-d_{t} e^{m-1}, d_{t} e^{m}\right)+2 k a_{\Omega}\left(d_{t} e^{m}, d_{t} e^{m}\right) \\
& =-\frac{1}{k}\left[a_{\Gamma}\left(u_{h}^{m}-u^{h, m-1}, 2 k d_{t} e^{m}\right)-a_{\Gamma}\left(u_{h}^{m-1}-u^{h, m-2}, 2 k d_{t} e^{m}\right)\right] \\
& =-2 a_{\Gamma}\left(u_{h}^{m}-u^{h, m-1}-u_{h}^{m-1}+u^{h, m-2}, d_{t} e^{m}\right) \\
& =2 a_{\Gamma}\left(d_{t} e^{m}, k d_{t} u_{h}^{m}-k d_{t} u^{h, m-1}\right) \\
& =2 a_{\Gamma}\left(d_{t} e^{m}, k d_{t} u_{h}^{m}-k d_{t} u_{h}^{m-1}+k d_{t} u_{h}^{m-1}-k d_{t} u^{h, m-1}\right) \\
& =2 a_{\Gamma}\left(d_{t} e^{m}, k^{2} d_{t t} u_{h}^{m}+k d_{t} e^{m-1}\right)-2 k a_{\Gamma}\left(d_{t} e^{m}, d_{t} e^{m}\right) \\
& =2 k^{2} a_{\Gamma}\left(d_{t} e^{m}, d_{t t}\left(u_{h}^{m}-e^{m}\right)\right) \\
& \leq 2 k^{2}\left[\frac{1}{4 k}\left\|d_{t} e^{m}\right\|_{W}^{2}+\tilde{C} k h^{-1}\left\|d_{t t}\left(e^{m}-u_{h}^{m}\right)\right\|_{0}^{2}\right] \\
& \leq \frac{1}{2} k\left\|d_{t} e^{m}\right\|_{W}^{2}+\frac{1}{2} k^{2}\left(\left\|d_{t t} e^{m}\right\|_{0}^{2}+\left\|d_{t t} u_{h}^{m}\right\|_{0}^{2}\right)
\end{aligned}
$$

where the last inequality follows from a properly chosen constant $C$ in (3.38). Similarly, the left hand side of (3.52) can be written as

$$
\begin{aligned}
\left\|d_{t} e^{m}\right\|_{0}^{2}-\left\|d_{t} e^{m-1}\right\|_{0}^{2}+k^{2}\left\|d_{t t} e^{m}\right\|_{0}^{2}+2 k\left\|d_{t} e^{m}\right\|_{W}^{2} \\
+2 n k \sum_{i=1}^{d-1} \int_{\Gamma} \frac{\alpha}{\sqrt{\boldsymbol{\tau}_{i} \cdot \mathbf{K} \boldsymbol{\tau}_{i}}}\left(d_{t} \mathbf{e}^{m} \cdot \boldsymbol{\tau}_{i}\right)^{2}
\end{aligned}
$$

Therefore,

$$
\left\|d_{t} e^{m}\right\|_{0}^{2}-\left\|d_{t} e^{m-1}\right\|_{0}^{2} \leq \frac{1}{2} k^{2}\left\|d_{t t} u_{h}^{m}\right\|_{0}^{2}
$$

which leads to

$$
\left\|d_{t} e^{m}\right\|_{0}^{2} \leq \frac{1}{2} k\left(\cdot k \sum_{j=2}^{m}\left\|d_{t t} u_{h}^{m}\right\|_{0}^{2}\right)+\left\|d_{t} e^{1}\right\|_{0}^{2}
$$

Note that

$$
\left\|d_{t} e^{1}\right\|_{0}^{2}=\left\|\frac{e^{1}-e^{0}}{k}\right\|_{0}^{2}=\frac{1}{k^{2}}\left\|e^{1}\right\|_{0}^{2}
$$

From (3.46), we have

$$
\left\|e^{1}\right\|_{0}^{2} \lesssim k^{2}\left(k\left\|d_{t} u_{h}^{1}\right\|_{W}^{2}\right) \lesssim k^{3}
$$

by using (3.37). Thus,

$$
\left\|d_{t} e^{1}\right\|_{0}^{2} \lesssim k
$$


So, from inequalities (3.39), (3.53) and (3.54), we have the estimate

$$
\left\|d_{t} e^{m}\right\|_{0} \lesssim k^{1 / 2} .
$$

Now we are ready to prove estimate (3.42). Taking $v_{h}=\left(\mathbf{v}_{h}, 0\right)$ in (3.35), we have

$$
n\left(\frac{\mathbf{e}^{m}-\mathbf{e}^{m-1}}{k}, \mathbf{v}_{h}\right)+a_{\Omega_{f}}\left(\mathbf{e}^{m}, \mathbf{v}_{h}\right)+a_{\Gamma}\left(u_{h}^{m}-u^{h, m-1}, v_{h}\right)+b\left(v_{h}, \eta^{m}\right)=0 .
$$

Therefore,

$$
\begin{aligned}
b\left(v_{h}, \eta^{m}\right) & \lesssim\left\|\nabla \mathbf{v}_{h}\right\|_{0}\left(\left\|d_{t} \mathbf{e}^{m}\right\|_{0}+\left\|\nabla \mathbf{e}^{m}\right\|_{0}\right)+\left|a_{\Gamma}\left(u_{h}^{m}-u^{h, m-1}, v_{h}\right)\right| \\
& =\left\|\nabla \mathbf{v}_{h}\right\|_{0}\left(\left\|d_{t} \mathbf{e}^{m}\right\|_{0}+\left\|\nabla \mathbf{e}^{m}\right\|_{0}\right)+\left|a_{\Gamma}\left(k d_{t} u_{h}^{m}+e^{m-1}, v_{h}\right)\right| \\
& \lesssim\left\|\nabla \mathbf{v}_{h}\right\|_{0}\left(\left\|d_{t} \mathbf{e}^{m}\right\|_{0}+\left\|\nabla \mathbf{e}^{m}\right\|_{0}+k\left\|\nabla d_{t} \varphi_{h}^{m}\right\|_{0}+\left\|\nabla \xi^{m-1}\right\|_{0}\right) .
\end{aligned}
$$

The discrete inf-sup condition (2.1) then implies

$$
\begin{aligned}
\left\|\eta^{m}\right\|_{0} & \lesssim\left\|d_{t} \mathbf{e}^{m}\right\|_{0}+\left\|\nabla \mathbf{e}^{m}\right\|_{0}+k\left\|\nabla d_{t} \varphi_{h}^{m}\right\|_{0}+\left\|\nabla \xi^{m-1}\right\|_{0} \\
& \leq\left\|d_{t} e^{m}\right\|_{0}+\left\|\nabla e^{m}\right\|_{0}+k\left\|\nabla d_{t} u_{h}^{m}\right\|_{0}+\left\|\nabla e^{m-1}\right\|_{0},
\end{aligned}
$$

or equivalently,

$$
\left\|\eta^{m}\right\|_{0}^{2} \lesssim\left(\left\|\nabla e^{m}\right\|_{0}^{2}+\left\|\nabla e^{m-1}\right\|_{0}^{2}+\left\|d_{t} e^{m}\right\|_{0}^{2}\right)+k^{2}\left\|\nabla d_{t} u_{h}^{m}\right\|_{0}^{2} .
$$

Estimate (3.42) then follows from (3.41), (3.55) and (3.37). This completes the proof of the theorem.

Combining Theorem 2 with Theorem [1] we have the following error estimates for DBES.

Corollary 1. Under the assumption (3.38), for $m=1, \ldots, J$, the following estimates hold:

$$
\begin{aligned}
& \left\|u^{h, m}-u\left(t_{m}\right)\right\|_{0} \lesssim k+h^{2}, \\
& \left\|\nabla\left(u^{h, m}-u\left(t_{m}\right)\right)\right\|_{0} \lesssim k^{1 / 2}+h, \\
& \left\|p_{f}^{h, m}-p_{f}\left(t_{m}\right)\right\|_{0} \lesssim k^{1 / 2}+h .
\end{aligned}
$$

Note that we have imposed the condition $k=O(h)$ in (3.38) for the error analysis of DBES, which is usually satisfied in practical computation in order to balance the accuracy in temporal and spacial approximations. In contrast, the fully explicit Euler scheme, although automatically decoupled, is subject to a severe stability condition $k=O\left(h^{2}\right)$ as usual.

\section{Numerical EXPERIMENTS}

For illustrating the convergence performance and mechanism, we report on our numerical experiments for both coupled and decoupled marching algorithms CBES and DBES.

Let the computational domain $\Omega$ be composed of $\Omega_{f}=(0,1) \times(1,2)$ and $\Omega_{p}=$ $(0,1) \times(0,1)$ with the interface $\Gamma=(0,1) \times\{1\}$. In the first testing problem, all the physical parameters $n, \rho, g, \nu, K, S_{0}$ and $\alpha$ are simply set to 1 . The Dirichlet boundary conditions are imposed for velocity $\mathbf{u}_{f}$ on $\partial \Omega_{f} \backslash \Gamma$ and for the piezometric 
head $\varphi$ on $\partial \Omega_{p} \backslash \Gamma$. The initial conditions, boundary conditions, and the forcing terms are chosen such that the exact solution is given by

$$
\begin{aligned}
\mathbf{u}_{f}(\mathbf{x}, t) & =\left(\left[x^{2}(y-1)^{2}+y\right] \cos t,\left[-\frac{2}{3} x(y-1)^{3}+2-\pi \sin (\pi x)\right] \cos t\right), \\
p_{f}(\mathbf{x}, t) & =[2-\pi \sin (\pi x)] \sin \left(\frac{\pi}{2} y\right) \cos t \\
\varphi(\mathbf{x}, t) & =[2-\pi \sin (\pi x)][1-y-\cos (\pi y)] \cos t .
\end{aligned}
$$

The finite element spaces are constructed by using the well-known MINI elements for the fluid flow and the linear Lagrangian elements for the porous media flow. In the implementation of the algebraic solver for CBES, an augmented Lagrangian algorithm (see II.5.2 in [4) is used to solve the saddle-point problem iteratively for $p_{f}$, and at each iteration the MATLAB GMRES routine is used to solve a coupled system for $\mathbf{u}_{f}$ and $\varphi$. For DBES, a conjugate gradient algorithm (see (21.2)-(21.12) in [10]) is applied in the Stokes solver. Furthermore, the MATLAB PCG routine with the sparse incomplete Cholesky factorization preconditioner is applied in various places of the implementation whenever an SPD problem is to be solved. The tolerance $\varepsilon$ is set to be $10^{-8}$ for the stopping criteria of the pressure iteration in both the augmented Lagrangian algorithm and the conjugate gradient algorithm. The tolerance and the maximum number of iterations in the MATLAB GMRES and PCG routines are set to be $10^{-10}$ and 1000, respectively.

We focus on the decoupled algorithm DBES. To demonstrate its convergence, we list in Table 1 and Table 2 the errors between the computed solution and the exact solution with varying spacing $h$ and time step $k$.

To examine the orders of convergence with respect to the spacing $h$, let us study the errors with a fixed time step $k=0.01$ and varying spacing $h=1 / 2,1 / 4,1 / 8$, $1 / 16,1 / 32$ by a mid-point mesh refinement. With such a small time step, observe from Table 2 that the approximation errors $O\left(k^{\gamma}\right)+O\left(h^{\mu}\right)$ are dominated by the $h$-terms $O\left(h^{\mu}\right)$ when $h$ varies in the given range. So, the ratio $\rho_{h,(\cdot)}^{l}$ of the approximation errors on two adjacent mesh levels $\Omega_{2 h}$ and $\Omega_{h}$ is approximately $(2 h)^{\mu} / h^{\mu}=2^{\mu}$, where $(\cdot)$ refers to $\mathbf{u}_{f}, \varphi$ and $p_{f}$, and $l$ refers to the $H^{l}$-error norm. Therefore, we can simply use $\rho_{h,(\cdot)}^{l}$ to estimate the corresponding order of convergence with respect to $h$. It is clearly seen from Table 3 that the error estimates of $u$ in Corollary 1 for the orders of convergence in space agree with the numerical experiments. It is also interesting to observe that the numerical rates for the Stokes pressure in $L^{2}$ seem to be better than the theoretical rates (3.58).

We take a more accurate, but slightly more sophisticated, approach to examine the orders of convergence with respect to the time step $k$ because the approximation errors $O\left(k^{\gamma}\right)+O\left(h^{\mu}\right)$ are dominated by the $h$-terms $O\left(h^{\mu}\right)$. For instance, assuming

$$
v^{h, k}\left(\mathbf{x}, t_{m}\right) \approx v\left(\mathbf{x}, t_{m}\right)+C_{1}\left(\mathbf{x}, t_{m}\right) k^{\gamma}+C_{2}\left(\mathbf{x}, t_{m}\right) h^{\mu},
$$

it follows that

$$
v^{h, k}\left(\mathbf{x}, t_{m}\right)-v^{h, \frac{1}{2} k}\left(\mathbf{x}, t_{m}\right) \approx C_{1}\left(\mathbf{x}, t_{m}\right)\left(1-1 / 2^{\gamma}\right) k^{\gamma} .
$$

Thus,

$$
\frac{v^{h, k}\left(\mathbf{x}, t_{m}\right)-v^{h, \frac{1}{2} k}\left(\mathbf{x}, t_{m}\right)}{v^{h, \frac{1}{2} k}\left(\mathbf{x}, t_{m}\right)-v^{h, \frac{1}{4} k}\left(\mathbf{x}, t_{m}\right)} \approx \frac{\left(1-1 / 2^{\gamma}\right)}{\left(1 / 2^{\gamma}-1 / 4^{\gamma}\right)}=\frac{\left(4^{\gamma}-2^{\gamma}\right)}{\left(2^{\gamma}-1\right)}
$$


TABLE 1. The convergence performance of DBES at time $t_{m}=0.5$ and $t_{m}=1$, with varying spacing $h$ but fixed time step $k=0.01$.

\begin{tabular}{|l|l|l|l|l|l|}
\hline \multicolumn{6}{|l|}{$m=50, t_{m}=0.5$} \\
\hline$h$ & $\left\|\mathbf{u}_{f}^{h, m}-\mathbf{u}_{f}\left(t_{m}\right)\right\|_{0}$ & $\left\|\varphi^{h, m}-\varphi\left(t_{m}\right)\right\|_{0}$ & $\left\|\nabla\left(\mathbf{u}_{f}^{h, m}-\mathbf{u}_{f}\left(t_{m}\right)\right)\right\|_{0}$ & $\left\|\nabla\left(\varphi^{h, m}-\varphi\left(t_{m}\right)\right)\right\|_{0}$ & $\left\|p_{f}^{h, m}-p_{f}\left(t_{m}\right)\right\|_{0}$ \\
\hline$\frac{1}{2}$ & 0.4875596461 & 0.4041223472 & 2.7803737315 & 2.5711752411 & 5.1011732529 \\
\hline$\frac{1}{4}$ & 0.1276607993 & 0.1218562903 & 1.4232574524 & 1.4941463864 & 1.5844129997 \\
\hline$\frac{1}{8}$ & 0.0323167897 & 0.0332595996 & 0.7162613241 & 0.7810566553 & 0.4385935708 \\
\hline$\frac{1}{16}$ & 0.0080998243 & 0.0086674998 & 0.3587345572 & 0.3952267936 & 0.1232022733 \\
\hline$\frac{1}{32}$ & 0.0020227613 & 0.0023358997 & 0.1794346920 & 0.1982265710 & 0.0365262409 \\
\hline \hline$m=100, t_{m}=1$ & \multicolumn{5}{|l|}{} \\
\hline$h$ & $\left\|\mathbf{u}_{f}^{h, m}-\mathbf{u}_{f}\left(t_{m}\right)\right\|_{0}$ & $\left\|\varphi^{h, m}-\varphi\left(t_{m}\right)\right\|_{0}$ & $\left\|\nabla\left(\mathbf{u}_{f}^{h, m}-\mathbf{u}_{f}\left(t_{m}\right)\right)\right\|_{0}$ & $\left\|\nabla\left(\varphi^{h, m}-\varphi\left(t_{m}\right)\right)\right\|_{0}$ & $\left\|p_{f}^{h, m}-p_{f}\left(t_{m}\right)\right\|_{0}$ \\
\hline$\frac{1}{2}$ & 0.3003203211 & 0.2531915482 & 1.7107457406 & 1.5828559416 & 3.0509756452 \\
\hline$\frac{1}{4}$ & 0.0786219134 & 0.0770174402 & 0.8760902638 & 0.9197305950 & 0.9577316927 \\
\hline$\frac{1}{8}$ & 0.0198994124 & 0.0212129034 & 0.4409560929 & 0.4808453403 & 0.2670986466 \\
\hline$\frac{1}{16}$ & 0.0049783640 & 0.0056816710 & 0.2208599688 & 0.2433352851 & 0.0755399068 \\
\hline$\frac{1}{32}$ & 0.0012381307 & 0.0017059304 & 0.1104782600 & 0.1220700067 & 0.0226324584 \\
\hline
\end{tabular}

TABLE 2. The convergence performance of DBES at time $t_{m}=$ 0.3 , with varying time step $k$ but fixed spacing $h=\frac{1}{16}$.

\begin{tabular}{|l||l|l|l|l|l|}
\hline$k$ & $\left\|\mathbf{u}_{f}^{h, m}-\mathbf{u}_{f}\left(t_{m}\right)\right\|_{0}$ & $\left\|\varphi^{h, m}-\varphi\left(t_{m}\right)\right\|_{0}$ & $\left\|\nabla\left(\mathbf{u}_{f}^{h, m}-\mathbf{u}_{f}\left(t_{m}\right)\right)\right\|_{0}$ & $\left\|\nabla\left(\varphi^{h, m}-\varphi\left(t_{m}\right)\right)\right\|_{0}$ & $\left\|p_{f}^{h, m}-p_{f}\left(t_{m}\right)\right\|_{0}$ \\
\hline$\frac{3}{50}$ & 0.0088334863 & 0.0099197352 & 0.3905397205 & 0.4302571249 & 0.1364831001 \\
\hline$\frac{3}{100}$ & 0.0088233887 & 0.0095499662 & 0.3905254667 & 0.4302439712 & 0.1350441304 \\
\hline$\frac{3}{200}$ & 0.0088214205 & 0.0093615439 & 0.3905211484 & 0.4302456417 & 0.1344419393 \\
\hline$\frac{3}{400}$ & 0.0088212220 & 0.0092669141 & 0.3905197280 & 0.4302489054 & 0.1341705746 \\
\hline$\frac{3}{800}$ & 0.0088213192 & 0.0092195656 & 0.3905192064 & 0.4302511923 & 0.1340423600 \\
\hline$\frac{3}{1600}$ & 0.0088214171 & 0.0091958923 & 0.3905189930 & 0.4302525056 & 0.1339801286 \\
\hline
\end{tabular}

TABLE 3. Examining the orders of convergence $O\left(h^{\mu}\right)$ for DBES by $\rho_{h,(\cdot)}^{l} \approx 2^{\mu}$, with varying spacing $h$, fixed time step $k=0.01$, and at time $t_{m}=0.5$.

\begin{tabular}{|l||l|l||l|l||l|l|}
\hline$h$ & $\left\|\mathbf{u}_{f}^{h, m}-\mathbf{u}_{f}\left(t_{m}\right)\right\|_{0}$ & $\rho_{h, \mathbf{u}_{f}}^{0}$ & $\left\|\varphi^{h, m}-\varphi\left(t_{m}\right)\right\|_{0}$ & $\rho_{h, \varphi}^{0}$ & $\left\|p_{f}^{h, m}-p_{f}\left(t_{m}\right)\right\|_{0}$ & $\rho_{h, p_{f}}^{0}$ \\
\hline$\frac{1}{2}$ & 0.4875596461 & 3.819181 & 0.4041223472 & 3.316385 & 5.1011732529 & 3.219598 \\
\hline$\frac{1}{4}$ & 0.1276607993 & 3.950293 & 0.1218562903 & 3.663793 & 1.5844129997 & 3.612486 \\
\hline$\frac{1}{8}$ & 0.0323167897 & 3.989814 & 0.0332595996 & 3.837277 & 0.4385935708 & 3.559947 \\
\hline$\frac{1}{16}$ & 0.0080998243 & 4.004340 & 0.0086674998 & 3.710562 & 0.1232022733 & 3.372980 \\
\hline$\frac{1}{32}$ & 0.0020227613 & & 0.0023358997 & & 0.0365262409 & \\
\hline \hline$h$ & $\left\|\nabla\left(\mathbf{u}_{f}^{h, m}-\mathbf{u}_{f}\left(t_{m}\right)\right)\right\|_{0}$ & $\rho_{h, \mathbf{u}_{f}}^{\frac{1}{2}}$ & $\left\|\nabla\left(\varphi^{h, m}-\varphi\left(t_{m}\right)\right)\right\|_{0}$ & $\rho_{h, \varphi}^{1}$ & & \\
\hline$\frac{1}{2}$ & 2.7803737315 & 1.953528 & 2.5711752411 & 1.720832 & & \\
\hline$\frac{1}{4}$ & 1.4232574524 & 1.987065 & 1.4941463864 & 1.912981 & & \\
\hline$\frac{1}{8}$ & 0.7162613241 & 1.996633 & 0.7810566553 & 1.976224 & & \\
\hline$\frac{1}{16}$ & 0.3587345572 & 1.999249 & 0.3952267936 & 1.993813 & & \\
\hline$\frac{1}{32}$ & 0.1794346920 & & 0.1982265710 & & & \\
\hline
\end{tabular}


TABLE 4. Examining the orders of convergence $O\left(k^{\gamma}\right)$ for DBES by $\rho_{k, v} \approx \frac{\left(4^{\gamma}-2^{\gamma}\right)}{\left(2^{\gamma}-1\right)}$, with varying time step $k$, fixed spacing $h=\frac{1}{8}$, and at time $t_{m}=1$.

\begin{tabular}{|l||l|l||l|l||l|l|}
\hline$k$ & $\left\|\mathbf{u}_{f}^{h, k}-\mathbf{u}_{f}^{h, \frac{1}{2} k}\right\|_{0}$ & $\rho_{k, \mathbf{u}_{f}}$ & $\left\|\varphi^{h, k}-\varphi^{h, \frac{1}{2} k}\right\|_{0}$ & $\rho_{k, \varphi}$ & $\left\|p_{f}^{h, k}-p_{f}^{h, \frac{1}{2} k}\right\|_{0}$ & $\rho_{k, p_{f}}$ \\
\hline$\frac{1}{10}$ & 0.0002137289 & 1.968292 & 0.0012999694 & 1.872289 & 0.0104060674 & 2.014106 \\
\hline$\frac{1}{20}$ & 0.0001085860 & 1.984729 & 0.0006943209 & 1.937976 & 0.0051665942 & 2.007383 \\
\hline$\frac{1}{40}$ & 0.0000547107 & 1.992313 & 0.0003582712 & 1.969515 & 0.0025737955 & 2.003703 \\
\hline$\frac{1}{80}$ & 0.0000274609 & 1.996049 & 0.0001819084 & 1.984896 & 0.0012845198 & 2.001851 \\
\hline$\frac{1}{160}$ & 0.0000137576 & & 0.0000916463 & & 0.0006416659 & \\
\hline \hline$k$ & $\left\|\nabla\left(\mathbf{u}_{f}^{h, k}-\mathbf{u}_{f}^{h, \frac{1}{2} k}\right)\right\|_{0}$ & $\rho_{k}, \nabla \mathbf{u}_{f}$ & $\left\|\nabla\left(\varphi^{h, k}-\varphi^{h, \frac{1}{2} k}\right)\right\|_{0}$ & $\rho_{k, \nabla}$ & & \\
\hline$\frac{1}{10}$ & 0.0028232766 & 1.943890 & 0.0067171597 & 1.860649 & & \\
\hline$\frac{1}{20}$ & 0.0014523847 & 1.971827 & 0.0036101161 & 1.933205 & & \\
\hline$\frac{1}{40}$ & 0.0007365681 & 1.985720 & 0.0018674255 & 1.967326 & & \\
\hline$\frac{1}{80}$ & 0.0003709326 & 1.992739 & 0.0009492203 & 1.983841 & & \\
\hline$\frac{1}{160}$ & 0.0001861421 & & 0.0004784760 & & & \\
\hline
\end{tabular}

Therefore, if we define

$$
\rho_{k, v}=\frac{\left\|v^{h, k}-v^{h, \frac{1}{2} k}\right\|_{0}}{\left\|v^{h, \frac{1}{2} k}-v^{h, \frac{1}{4} k}\right\|_{0}}
$$

where $v=\mathbf{u}_{f}, \varphi, p_{f}, \nabla \mathbf{u}_{f}$ or $\nabla \varphi$, then $\rho_{k, v} \approx \frac{\left(4^{\gamma}-2^{\gamma}\right)}{\left(2^{\gamma}-1\right)}$ from (4.6). In particular, $\rho_{k, v} \approx 2$ for $\gamma=1$ when the corresponding order of convergence in time is of $O(k)$. A set of values of $\rho_{k, v}$ are listed in Table 4 with varying time step $k$, but a fixed spacing $h=\frac{1}{8}$, which clearly suggests that the concerned orders of convergence in time are all of $O(k)$ for DBES. To further confirm this same observation, we also list in Table 5 another set of values of $\rho_{k, v}$ with varying time step $k$, but a different spacing $h=\frac{1}{16}$ for DBES. Therefore, the numerical experiments strongly suggest that the orders of convergence in time in the error estimates (3.57) and (3.58) for the $H^{1}$-norm of $u$ and the $L^{2}$-norm of pressure $p_{f}$ might not be optimal for DBES, and may be further improved from $O\left(k^{1 / 2}\right)$ to $O(k)$ by a finer analysis. However, the error estimate (3.56) for the $L^{2}$-norm of $u$ is optimal in both time and space for DBES.

Finally, for comparison, we list in Table 6 the exact solution and the solutions for CBES and DBES at selected locations, with varying spacing $h$ from $1 / 2$ to $1 / 16$ and with a fixed time step $k=0.01$. It is seen that DBES almost retains the same accuracy as CBES, and the approximation accuracy does not deteriorate due to the decoupling technique.

It is also of practical interest to examine the effects of the physical parameters. For illustration, we consider another test problem with the varying hydraulic conductivity $K$, while other parameters $n, \rho, g, \nu, S_{0}$ and $\alpha$, as well as the computational domain and boundary conditions, remain the same as in the previous test problem. However, the initial data, boundary data, and the forcing terms are 
TABLE 5. Examining the orders of convergence $O\left(k^{\gamma}\right)$ for DBES by $\rho_{k, v} \approx \frac{\left(4^{\gamma}-2^{\gamma}\right)}{\left(2^{\gamma}-1\right)}$, with varying time step $k$, fixed spacing $h=\frac{1}{16}$, and at time $t_{m}=0.6$.

\begin{tabular}{|l||l|l||l|l||l|l|}
\hline$k$ & $\left\|\mathbf{u}_{f}^{h, k}-\mathbf{u}_{f}^{h, \frac{1}{2} k}\right\|_{0}$ & $\rho_{k, \mathbf{u}_{f}}$ & $\left\|\varphi^{h, k}-\varphi^{h, \frac{1}{2} k}\right\|_{0}$ & $\rho_{k, \varphi}$ & $\left\|p_{f}^{h, k}-p_{f}^{h, \frac{1}{2} k}\right\|_{0}$ & $\rho_{k, p_{f}}$ \\
\hline$\frac{3}{50}$ & 0.0001424214 & 1.967334 & 0.0005874511 & 1.874202 & 0.0066395100 & 2.002696 \\
\hline$\frac{3}{100}$ & 0.0000723931 & 1.987050 & 0.0003134407 & 1.937613 & 0.0033152857 & 2.002180 \\
\hline$\frac{3}{200}$ & 0.0000364325 & 1.994091 & 0.0001617664 & 1.969082 & 0.0016558376 & 2.001300 \\
\hline$\frac{3}{400}$ & 0.0000182702 & & 0.0000821532 & & 0.0008273811 & \\
\hline \hline$k$ & $\left\|\nabla\left(\mathbf{u}_{f}^{h, k}-\mathbf{u}_{f}^{h, \frac{1}{2} k}\right)\right\|_{0}$ & $\rho_{k}, \nabla \mathbf{u}_{f}$ & $\left\|\nabla\left(\varphi^{h, k}-\varphi^{h, \frac{1}{2} k}\right)\right\|_{0}$ & $\rho_{k, \nabla \varphi}$ & & \\
\hline$\frac{3}{50}$ & 0.0017126004 & 1.966713 & 0.0030124037 & 1.860959 & & \\
\hline$\frac{3}{100}$ & 0.0008707930 & 1.985332 & 0.0016187370 & 1.932211 & & \\
\hline$\frac{3}{200}$ & 0.0004386133 & 1.993384 & 0.0008377642 & 1.966645 & & \\
\hline$\frac{3}{400}$ & 0.0002200345 & & 0.0004259866 & & & \\
\hline
\end{tabular}

TABLE 6 . The comparison between the exact solution and the solutions of CBES and DBES at the point $\mathbf{x}=(0.5,1.5)$ for the fluid flow and at $(0.5,0.5)$ for the porous medium flow with a fixed time step $k=0.01$, at $t_{m}=0.1$.

\begin{tabular}{|c|c|c|c|c|c|c|}
\hline$h$ & \multicolumn{2}{|c|}{$\mathbf{u}_{f}\left(\mathbf{x}, t_{m}\right)$} & \multicolumn{2}{|c|}{$\mathbf{u}_{f h}^{m}$} & \multicolumn{2}{|c|}{$\mathbf{u}_{f}^{h, m}$} \\
\hline$\frac{1}{2}$ & \multicolumn{2}{|c|}{$(1.5546940082,-1.1773479523)$} & \multicolumn{2}{|c|}{$(1.5921313881,-1.1322728727)$} & \multicolumn{2}{|c|}{$(1.5921313884,-1.1322728717)$} \\
\hline$\frac{1}{4}$ & \multicolumn{2}{|c|}{$(1.5546940082,-1.1773479523)$} & \multicolumn{2}{|c|}{$(1.5593652198,-1.1636481793)$} & \multicolumn{2}{|c|}{$(1.5593709738,-1.1636555301)$} \\
\hline$\frac{1}{8}$ & \multicolumn{2}{|c|}{$(1.5546940082,-1.1773479523)$} & \multicolumn{2}{|c|}{$(1.5564707718,-1.1741917982)$} & \multicolumn{2}{|c|}{$(1.5564804765,-1.1741981061)$} \\
\hline$\frac{1}{16}$ & \multicolumn{2}{|c|}{$(1.5546940082,-1.1773479523)$} & \multicolumn{2}{|c|}{$(1.5551444509,-1.1765370209)$} & \multicolumn{2}{|c|}{$(1.5551472727,-1.1765402623)$} \\
\hline$h$ & $p_{f}\left(\mathbf{x}, t_{m}\right)$ & $p_{f h}^{m}$ & $p_{f}^{h, m}$ & $\varphi\left(\mathbf{x}, t_{m}\right)$ & $\varphi_{h}^{m}$ & $\varphi^{h, m}$ \\
\hline$\frac{1}{2}$ & -0.8031951295 & 0.1664526584 & 0.1642503932 & -0.5679447227 & -0.5423730106 & -0.5421249643 \\
\hline$\frac{1}{4}$ & -0.8031951295 & -1.1664301192 & -1.1673319397 & -0.5679447227 & -0.5701236315 & -0.5700535315 \\
\hline$\frac{1}{8}$ & -0.8031951295 & -0.8840872577 & -0.8845656184 & -0.5679447227 & -0.5690320401 & -0.5690082459 \\
\hline$\frac{1}{16}$ & -0.8031951295 & -0.8183287665 & -0.8186986757 & -0.5679447227 & -0.5681775839 & -0.5681655349 \\
\hline
\end{tabular}

chosen such that the exact solution of the coupled model is given by

$$
\begin{aligned}
\mathbf{u}_{f}(\mathbf{x}, t) & =\left(\left(y^{2}-2 y+1\right) \cos t,\left(x^{2}-x\right) \cos t\right) \\
p_{f}(\mathbf{x}, t) & =\left[2 \nu(x+y-1)+\frac{g n}{3 K}\right] \cos t \\
\phi(\mathbf{x}, t) & =\left[\frac{n}{K}\left(x(1-x)(y-1)+\frac{1}{3} y^{3}-y^{2}+y\right)+\frac{2 \nu}{g} x\right] \cos t .
\end{aligned}
$$

The approximation errors listed in Tables 7 , 8 , and 9 corresponding to $K=$ $0.1,0.01,0.001$ show that the numerical solutions approximate the exact solution with satisfied order of convergence when the hydraulic conductivity $K$ varies. Note that Tables 17 8, and 9 are for absolute errors instead of relative errors, while the orders of magnitude for $p_{f}$ and $\phi$ increase as $K$ decreases. For more numerical experiments and simulation, the reader is referred to [5, 25, 26]. 
TABLE 7. The approximation errors for DBES for $K=0.1$ with varying spacing $h$, fixed time step $k=10^{-3}$, and at time $t_{m}=0.05$.

\begin{tabular}{|l||l|l|l|l|l|}
\hline$h$ & $\left\|\mathbf{u}_{f}^{h, m}-\mathbf{u}_{f}\left(t_{m}\right)\right\|_{0}$ & $\left\|\varphi^{h, m}-\varphi\left(t_{m}\right)\right\|_{0}$ & $\left\|p_{f}^{h, m}-p_{f}\left(t_{m}\right)\right\|_{0}$ & $\left\|\nabla\left(\mathbf{u}_{f}^{h, m}-\mathbf{u}_{f}\left(t_{m}\right)\right)\right\|_{0}$ & $\left\|\nabla\left(\varphi^{h, m}-\varphi\left(t_{m}\right)\right)\right\|_{0}$ \\
\hline$\frac{1}{2}$ & 0.0730762822 & 0.3481047236 & 0.2686044007 & 0.3998059670 & 2.7633839815 \\
\hline$\frac{1}{4}$ & 0.0183429476 & 0.0894437360 & 0.0707507409 & 0.1987595531 & 1.4254426719 \\
\hline$\frac{1}{8}$ & 0.0045923514 & 0.0225179093 & 0.0199145832 & 0.0991959428 & 0.7186942188 \\
\hline$\frac{1}{16}$ & 0.0011481794 & 0.0056387600 & 0.0058051380 & 0.0495634151 & 0.3601255888 \\
\hline$\frac{1}{32}$ & 0.0002868260 & 0.0014108989 & 0.0017768174 & 0.0247744729 & 0.1801616776 \\
\hline
\end{tabular}

TABLE 8. The approximation errors for DBES for $K=0.01$ with varying spacing $h$, fixed time step $k=10^{-3}$, and at time $t_{m}=0.05$.

\begin{tabular}{|l||l|l|l|l|l|}
\hline$h$ & $\left\|\mathbf{u}_{f}^{h, m}-\mathbf{u}_{f}\left(t_{m}\right)\right\|_{0}$ & $\left\|\varphi^{h, m}-\varphi\left(t_{m}\right)\right\|_{0}$ & $\left\|p_{f}^{h, m}-p_{f}\left(t_{m}\right)\right\|_{0}$ & $\left\|\nabla\left(\mathbf{u}_{f}^{h, m}-\mathbf{u}_{f}\left(t_{m}\right)\right)\right\|_{0}$ & $\left\|\nabla\left(\varphi^{h, m}-\varphi\left(t_{m}\right)\right)\right\|_{0}$ \\
\hline$\frac{1}{2}$ & 0.0730138376 & 3.4882311353 & 0.2799971333 & 0.4002664205 & 27.7449194208 \\
\hline$\frac{1}{4}$ & 0.0183265110 & 0.8938577440 & 0.0758389140 & 0.1988147132 & 14.2788409796 \\
\hline$\frac{1}{8}$ & 0.0045895877 & 0.2248207490 & 0.0226786588 & 0.0992007783 & 7.1902105579 \\
\hline$\frac{1}{16}$ & 0.0011476663 & 0.0562930252 & 0.0070463192 & 0.0495638447 & 3.6016449317 \\
\hline$\frac{1}{32}$ & 0.0002867438 & 0.0140869534 & 0.0018642987 & 0.0247745120 & 1.8016691717 \\
\hline
\end{tabular}

TABLE 9. The approximation errors for DBES for $K=0.001$ with varying spacing $h$, fixed time step $k=10^{-4}$, and at time $t_{m}=$ 0.005 .

\begin{tabular}{|l||l|l|l|l|l|}
\hline$h$ & $\left\|\mathbf{u}_{f}^{h, m}-\mathbf{u}_{f}\left(t_{m}\right)\right\|_{0}$ & $\left\|\varphi^{h, m}-\varphi\left(t_{m}\right)\right\|_{0}$ & $\left\|p_{f}^{h, m}-p_{f}\left(t_{m}\right)\right\|_{0}$ & $\left\|\nabla\left(\mathbf{u}_{f}^{h, m}-\mathbf{u}_{f}\left(t_{m}\right)\right)\right\|_{0}$ & $\left\|\nabla\left(\varphi^{h, m}-\varphi\left(t_{m}\right)\right)\right\|_{0}$ \\
\hline$\frac{1}{2}$ & 0.0730393023 & 34.9379941545 & 0.2992707690 & 0.4012939366 & 277.9462760134 \\
\hline$\frac{1}{4}$ & 0.0183334901 & 8.9502226078 & 0.0790647707 & 0.1991521538 & 143.0136301460 \\
\hline$\frac{1}{8}$ & 0.0045893020 & 2.2508374474 & 0.0209467869 & 0.0993355912 & 72.0030993422 \\
\hline$\frac{1}{16}$ & 0.0011477180 & 0.5635365671 & 0.0059432842 & 0.0496265430 & 36.0633380934 \\
\hline$\frac{1}{32}$ & 0.0002869416 & 0.1409357933 & 0.0018230784 & 0.0248053217 & 18.0393893493 \\
\hline
\end{tabular}

\section{Conclusions}

A decoupling approach based on interface approximation via temporal extrapolation is proposed for devising decoupled marching algorithms for the non-stationary mixed Stokes-Darcy model. An error analysis is presented and numerical experiments are conducted to demonstrate the computational effectiveness of the decoupling approach.

\section{APPENDIX}

Proof of Lemma 2. In order to prove the estimate (3.37), subtracting the coupled backward Euler scheme (2.10)-(2.11) on two adjacent time levels and noticing the definition of $d_{t} u_{h}^{0}$, we have for $m=1, \ldots, J$,

$$
\left(\frac{d_{t} u_{h}^{m}-d_{t} u_{h}^{m-1}}{k}, v_{h}\right)+a\left(d_{t} u_{h}^{m}, v_{h}\right)=\frac{1}{k}\left[f^{m}\left(v_{h}\right)-f^{m-1}\left(v_{h}\right)\right] \forall v_{h} \in V_{h} .
$$


Taking $v_{h}=2 k d_{t} u_{h}^{m}=2 k\left(d_{t} \mathbf{u}_{f h}^{m}, d_{t} \varphi_{h}^{m}\right) \in V_{h}$ in A.1), we obtain (A.2)

$$
\left(\frac{d_{t} u_{h}^{m}-d_{t} u_{h}^{m-1}}{k}, 2 k d_{t} u_{h}^{m}\right)+a\left(d_{t} u_{h}^{m}, 2 k d_{t} u_{h}^{m}\right)=\frac{1}{k}\left[f^{m}\left(2 k d_{t} u_{h}^{m}\right)-f^{m-1}\left(2 k d_{t} u_{h}^{m}\right)\right] .
$$

Then we have

$$
\begin{aligned}
& \left\|d_{t} u_{h}^{m}\right\|_{0}^{2}-\left\|d_{t} u_{h}^{m-1}\right\|_{0}^{2}+k^{2}\left\|d_{t t} u_{h}^{m}\right\|_{0}^{2}+2 k\left\|d_{t} u_{h}^{m}\right\|_{W}^{2} \\
& =n \frac{1}{k} \int_{\Omega_{f}}\left(\mathbf{g}_{f}^{m}-\mathbf{g}_{f}^{m-1}\right) \cdot\left(2 k d_{t} \mathbf{u}_{f h}^{m}\right)+\rho g \frac{1}{k} \int_{\Omega_{p}}\left(g_{p}^{m}-g_{p}^{m-1}\right)\left(2 k d_{t} \varphi_{h}^{m}\right) \\
& \quad-2 n k \sum_{i=1}^{d-1} \int_{\Gamma} \frac{\alpha}{\sqrt{\boldsymbol{\tau}_{i} \cdot \mathbf{K} \boldsymbol{\tau}_{i}}}\left(d_{t} \mathbf{u}_{f h}^{m} \cdot \boldsymbol{\tau}_{i}\right)^{2} \\
& \leq C^{2} n \nu^{-1} \int_{t_{m-1}}^{t_{m}}\left\|\mathbf{g}_{f t}(t)\right\|_{0}^{2} d t+n \nu k\left\|\nabla d_{t} \mathbf{u}_{f h}^{m}\right\|_{0}^{2} \\
& \quad+C^{2} \rho g K^{-1} \int_{t_{m-1}}^{t_{m}}\left\|g_{p t}(t)\right\|_{0}^{2} d t+\rho g K k\left\|\nabla d_{t} \varphi_{h}^{m}\right\|_{0}^{2} .
\end{aligned}
$$

Therefore,

$$
\begin{aligned}
& \left\|d_{t} u_{h}^{J}\right\|_{0}^{2}+k^{2} \sum_{m=1}^{J}\left\|d_{t t} u_{h}^{m}\right\|_{0}^{2}+k \sum_{m=1}^{J}\left\|d_{t} u_{h}^{m}\right\|_{W}^{2} \\
& \leq C^{2} n \nu^{-1} \int_{0}^{T}\left\|\mathbf{g}_{f t}(t)\right\|_{0}^{2} d t+C^{2} \rho g K^{-1} \int_{0}^{T}\left\|g_{p t}(t)\right\|_{0}^{2} d t+\left\|d_{t} u_{h}^{0}\right\|_{0}^{2},
\end{aligned}
$$

which yields estimate 3.37).

We now turn to prove (3.39). For $m=2, \ldots, J$, taking $v_{h}=2\left(d_{t} u_{h}^{m}-d_{t} u_{h}^{m-1}\right) \in$ $V_{h}$ in (A.1), we have

$$
\begin{aligned}
& 2 k\left(d_{t t} u_{h}^{m}, d_{t t} u_{h}^{m}\right)+2 a_{\Omega}\left(d_{t} u_{h}^{m}, d_{t} u_{h}^{m}-d_{t} u_{h}^{m-1}\right) \\
= & \frac{1}{k}\left[f^{m}\left(2\left(d_{t} u_{h}^{m}-d_{t} u_{h}^{m-1}\right)\right)-f^{m-1}\left(2\left(d_{t} u_{h}^{m}-d_{t} u_{h}^{m-1}\right)\right)\right] \\
& -2 a_{\Gamma}\left(d_{t} u_{h}^{m}, d_{t} u_{h}^{m}-d_{t} u_{h}^{m-1}\right) \\
= & 2\left[f^{m}\left(d_{t t} u_{h}^{m}\right)-f^{m-1}\left(d_{t t} u_{h}^{m}\right)\right]+2 k a_{\Gamma}\left(d_{t t} u_{h}^{m}, d_{t} u_{h}^{m-1}\right) .
\end{aligned}
$$

Then we have

$$
\begin{aligned}
& 2 k\left\|d_{t t} u_{h}^{m}\right\|_{0}^{2}+\left\|d_{t} u_{h}^{m}\right\|_{W}^{2}-\left\|d_{t} u_{h}^{m-1}\right\|_{W}^{2}+k^{2}\left\|d_{t t} u_{h}^{m}\right\|_{W}^{2} \\
& +n \sum_{i=1}^{d-1} \int_{\Gamma} \frac{\alpha}{\sqrt{\boldsymbol{\tau}_{i} \cdot \mathbf{K} \boldsymbol{\tau}_{i}}}\left(d_{t} \mathbf{u}_{f h}^{m} \cdot \boldsymbol{\tau}_{i}\right)^{2}-n \sum_{i=1}^{d-1} \int_{\Gamma} \frac{\alpha}{\sqrt{\boldsymbol{\tau}_{i} \cdot \mathbf{K} \boldsymbol{\tau}_{i}}}\left(d_{t} \mathbf{u}_{f h}^{m-1} \cdot \boldsymbol{\tau}_{i}\right)^{2} \\
& +n k^{2} \sum_{i=1}^{d-1} \int_{\Gamma} \frac{\alpha}{\sqrt{\boldsymbol{\tau}_{i} \cdot \mathbf{K} \boldsymbol{\tau}_{i}}}\left(d_{t t} \mathbf{u}_{f h}^{m} \cdot \boldsymbol{\tau}_{i}\right)^{2} \\
& \leq n \int_{t_{m-1}}^{t_{m}}\left\|\mathbf{g}_{f t}(t)\right\|_{0}^{2} d t+\rho g S_{0}^{-1} \int_{t_{m-1}}^{t_{m}}\left\|g_{p t}(t)\right\|_{0}^{2} d t+k\left\|d_{t t} u_{h}^{m}\right\|_{0}^{2} \\
& +k^{2}\left\|d_{t t} u_{h}^{m}\right\|_{W}^{2}+C^{2} n \rho g \nu^{-1} K^{-1}\left\|d_{t} u_{h}^{m-1}\right\|_{W}^{2} .
\end{aligned}
$$


Therefore,

$$
\left\|d_{t} u_{h}^{J}\right\|_{W}^{2}+k \sum_{m=2}^{J}\left\|d_{t t} u_{h}^{m}\right\|_{0}^{2} \leq n \int_{t_{1}}^{T}\left\|\mathbf{g}_{f t}(t)\right\|_{0}^{2} d t+\rho g S_{0}^{-1} \int_{t_{1}}^{T}\left\|g_{p t}(t)\right\|_{0}^{2} d t
$$

$$
+\sum_{m=1}^{J-1} C^{2} n \rho g \nu^{-1} K^{-1}\left\|d_{t} u_{h}^{m}\right\|_{W}^{2}+\left\|d_{t} u_{h}^{1}\right\|_{W}^{2}+C\left\|\nabla d_{t} \mathbf{u}_{f h}^{1}\right\|_{0}^{2}
$$

Note that

$$
\begin{aligned}
\left\|\nabla d_{t} \mathbf{u}_{f h}^{1}\right\|_{0}= & \frac{1}{k}\left\|\nabla\left(\mathbf{u}_{f h}^{1}-\mathbf{u}_{f h}^{0}\right)\right\|_{0} \\
= & \frac{1}{k}\left\|\nabla\left(\left[\mathbf{u}_{f h}^{1}-\mathbf{u}_{f h}^{0}\right]-\left[\mathbf{u}_{f h}\left(t_{1}\right)-\mathbf{u}_{f h}\left(t_{0}\right)\right]+\left[\mathbf{u}_{f h}\left(t_{1}\right)-\mathbf{u}_{f h}\left(t_{0}\right)\right]\right)\right\|_{0} \\
\leq & \frac{1}{k}\left[\left\|\nabla\left[\mathbf{u}_{f h}^{1}-\mathbf{u}_{f h}\left(t_{1}\right)\right]\right\|_{0}+\left\|\nabla\left[\mathbf{u}_{f h}^{0}-\mathbf{u}_{f h}\left(t_{0}\right)\right]\right\|_{0}\right. \\
& \left.\quad+\left\|\nabla\left[\int_{t_{0}}^{t_{1}} \frac{\partial}{\partial t} \mathbf{u}_{f h}(t) d t\right]\right\|_{0}\right] \\
\lesssim & +k^{-1} h+k^{-1} h+\max _{t_{0} \leq t \leq t_{1}}\left\|\frac{\partial}{\partial t} \nabla \mathbf{u}_{f h}(t)\right\|_{0} \lesssim C
\end{aligned}
$$

by using (2.9), (3.10) and the stability condition $k=O(h)$. Similarly, we also have $\left\|\nabla d_{t} \mathbf{u}_{f h}^{1}\right\|_{0} \lesssim C$. Estimate (3.39) then follows from (A.7) and (A.8) by using Gronwall's lemma, which completes the proof of Lemma 2 .

\section{ACKNOWLEDGEMENTS}

The authors would like to thank the editor and referees for their careful review and helpful comments and suggestions which substantially improved the manuscript.

\section{REFERENCES}

[1] T. Arbogast and D.S. Brunson, A computational method for approximating a Darcy-Stokes system governing a vuggy porous medium, Comput. Geosci. (2007), Vol. 11, pp. 207-218. MR2344200 (2009b:76155)

[2] J. Bear, Hydraulics of groundwater, McGraw-Hill, New York, 1979.

[3] G.S. Beavers and D. Joseph, Boundary conditions at a naturally permeable wall, J. Fluid Mech. (1967), Vol. 30, pp. 197-207.

[4] F. Brezzi and M. Fortin, Mixed and hybrid finite element methods, Springer-Verlag, 1991. MR.1115205 (92d:65187)

[5] M. Cai, M. Mu, and J. Xu, Decoupled preconditioners for Stokes-Darcy coupling problem with applications in porous media, submitted, 2007.

[6] M. Discacciati, Domain decomposition methods for the coupling of surface and groundwater flows, Ph.D. dissertation, École Polytechnique Fédérale de Lausanne, 2004.

[7] M. Discacciati, E. Miglio, and A. Quarteroni, Mathematical and numerical models for coupling surface and groundwater flows, Appl. Numer. Math (2002), Vol. 43, pp. 57-74. MR.1936102 (2003h:76087)

[8] M. Discacciati and A. Quarteroni, Convergence analysis of a subdomain iterative method for the finite element approximation of the coupling of Stokes and Darcy equations, Comput. Visual Sci. (2004), Vol. 6, pp. 93-103. MR2061270(2005e:65142)

[9] J. Galvis and M. Sarkis, Balancing domain decomposition methods for mortar coupling Stokes-Darcy Systems, Proceedings of the 16th International Conference on Domain Decomposition Methods, 2005. 
[10] R. Glowinshi, Finite element methods for incompressible viscous flow, Handbook of Numerical Analysis, Vol. IX, Numerical Methods for Fluids (Part 3), edited by P.G. Ciarlet and J.L. Lions, Elsevier Science B.V., 2003. MR2009826

[11] Y. He and W. Sun, Stability and convergence of the Crank-Nicolson/Adams-Bashforth scheme for the time-dependent Navier-Stokes equations, Siam J. Numer. Anal. (2007), Vol. 45, No. 2, pp. 837-869. MR2300299(2008d:65110)

[12] R. H. W. Hoppe, P. Porta, and Y. Vassilevski, Computational issues related to iterative coupling of subsurface and channel flows, Calcolo, Vol. 44, No. 1. (2007), pp. 1-20. MR2301278 (2008a:76088)

[13] W. Jäger and A. Mikelić, On the interface boundary condition of Beavers, Joseph, and Saffman, SIAM J. Appl. Math. (2000), Vol. 60, No. 4, pp. 1111-1127. MR1760028 (2001e:76122)

[14] W. Jäger, A. Mikelić, and N. Neuss, Asymptotic analysis of the laminar viscous flow over a porous bed, SIAM J. Sci. Comput. (2001), Vol. 22, No. 6, pp. 2006-2028. MR1856299 (2002f:76065)

[15] W.J. Layton, F. Schieweck, and I. Yotov, Coupling fluid flow with porous media flow, SIAM J. Numer. Anal. (2003), Vol. 40, No. 6, pp. 2195-2218. MR1974181 (2004c:76048)

[16] E. Miglio, A. Quarteroni, and F. Saleri, Coupling of free surface and groundwater flows, Computers Fluids (2003), Vol. 32, pp. 73-83.

[17] M. Mu, A Linearized Crank-Nicolson Galerkin Method for the Ginzburg-Landau Model, SIAM J. Sci. Comput. (1997), Vol. 18, pp. 1028-1039. MR1453555 (98f:82140)

[18] M. Mu and J. Xu, A two-grid method of a mixed Stokes-Darcy model for coupling fluid flow with porous media flow, SIAM J. Numer. Anal. (2007), Vol. 45, No. 5, pp. 1801-1813. MR2346360 (2008i:65264)

[19] L.E. Payne and B. Straughan, Analysis of the boundary condition at the interface between a viscous fluid and a porous medium and related modeling questions, J. Math. Pure Appl. (1998), Vol. 77, pp. 317-354. MR1623387 (99c:35200)

[20] B. Rivière and I. Yotov, Locally conservative coupling of Stokes and Darcy flows, SIAM J. Numer. Anal. (2005), Vol. 40, No. 5, pp. 1959-1977. MR2139232 (2006a:76035)

[21] P.G. Saffman, On the boundary condition at the interface of a porous medium, Studies in Applied Mathematics (1971), Vol. 1, No. 2, pp. 93-101.

[22] M.F. Wheeler, A priori $L_{2}$ error estimates for Galerkin approximations to parabolic partial differential equations, SIAM J. Numer. Anal. (1973), Vol. 10, pp. 723-759. MR0351124 (50:3613)

[23] W.L. Wood, Introduction to numerical methods for water resources, Oxford University Press, 1993. MR1231800 (94m:86001)

[24] J. Xu, Theory of multilevel methods, Ph.D. dissertation, Cornell University, Ithaca, NY, 1989.

[25] J. Xu, M. Mu, and M. Cai, Numerical Solution to a Mixed Navier-Stokes/Darcy Model by the Two-grid Approach, submitted, 2008.

[26] X. Zhu, Numerical methods for a mixed Stokes-Darcy model, Ph.D. dissertation, HKUST, Hong Kong, 2007.

Department of Mathematics, Hong Kong University of Science and Technology, Clearwater Bay, Kowloon, Hong Kong

E-mail address: mamu@ust.hk

Department of Mathematics, Hong Kong University of Science and Technology, Clearwater Bay, Kowloon, Hong Kong

E-mail address: xhzhu@ust.hk 